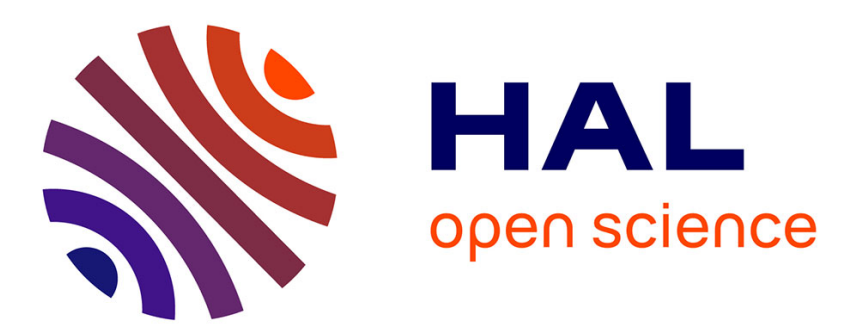

\title{
On the use of dynamic vibration absorbers to counteract the loss of sound insulation due to mass-spring-mass resonance effects in External Thermal Insulation Composite Systems
}

\author{
Nicolaas Bernardus B Roozen, D. Urbán, E.A. A Piana, C. Glorieux
}

\section{To cite this version:}

Nicolaas Bernardus B Roozen, D. Urbán, E.A. A Piana, C. Glorieux. On the use of dynamic vibration absorbers to counteract the loss of sound insulation due to mass-spring-mass resonance effects in External Thermal Insulation Composite Systems. Applied Acoustics, 2021, 178, pp.107999. 10.1016/j.apacoust.2021.107999 . hal-03202436

\section{HAL Id: hal-03202436 \\ https://hal.science/hal-03202436}

Submitted on 19 Apr 2021

HAL is a multi-disciplinary open access archive for the deposit and dissemination of scientific research documents, whether they are published or not. The documents may come from teaching and research institutions in France or abroad, or from public or private research centers.
L'archive ouverte pluridisciplinaire HAL, est destinée au dépôt et à la diffusion de documents scientifiques de niveau recherche, publiés ou non, émanant des établissements d'enseignement et de recherche français ou étrangers, des laboratoires publics ou privés. 


\title{
On the use of dynamic vibration absorbers to counteract the loss of sound insulation due to mass-spring-mass resonance effects in External Thermal Insulation Composite Systems.
}

\author{
N.B. Roozen ${ }^{\mathrm{a}}$, D. Urbán ${ }^{\mathrm{b}, \mathrm{c}}$, E.A. Piana ${ }^{\mathrm{d}}$, C. Glorieux ${ }^{\mathrm{a}}$ \\ ${ }^{a}$ Laboratory of Acoustics, Soft Matter and Biophysics, Department of Physics and Astronomy, KU Leuven, Celestijnenlaan 200D, 3001 \\ Leuven, Belgium. \\ ${ }^{b}$ Czech Technical University in Prague, Faculty of Electrical Engineering, Department of Physics, Technicka 2, 16627 Prague, Czech \\ Republic. \\ ${ }^{c}$ Slovak University of Technology in Bratislava, Faculty of Civil Engineering, Department of Materials Engineering and Physics, \\ Radlinského 11, 81005 Bratislava, Slovakia. \\ ${ }^{d}$ Department of Mechanical and Industrial Engineering, University of Brescia, 25123 Brescia, Italy.
}

\begin{abstract}
One of the common ways to increase the energy performance of existing buildings is to add a thermal insulation layer to their façade elements, which in turn is covered by a thin protecting cement plaster. Such an arrangement is commonly known as External Thermal Insulation Composite System (ETICS). This article studies the effect of an ETICS implementation induced mechanical resonance mechanism, which results in a loss of performance in the sound insulation spectrum. A mitigating approach is proposed, which is based on an array of dynamic vibration absorbers that were designed to counteract the decrease of the sound reduction index. Using a wavenumber domain approach combined with unit cell FEM-modelling employing Bloch-Floquet boundary conditions, numerical predictions are presented for the sound reduction index. A number of different dynamic vibration absorber designs were tested on a silicate cement brick wall in a transmission loss facility. The predictions are in good agreement with experimental data obtained by classical, microphone based acoustic isolation measurements and laser Doppler vibrometry.
\end{abstract}

Keywords: ETICS, sound reduction index, mass-spring-mass resonance, locally resonant metamaterials, FE method, laser Doppler vibrometry

\section{Introduction}

Environmental protection and reduction of energy use are among the main issues to tackle on the roadmap towards a sustainable future. European directive 2010/31/UE on the energy performance of buildings states that edifices account for $40 \%$ of total energy consumption in the European Union (EU). Therefore the energy performance of buildings needs to be enhanced. A common way to improve the thermal insulation of building façades is the use of an External Thermal Insulation Composite System (ETICS), which basically consists in adding a layer of thermal insulation material (e.g. expanded polystyrene) to the base wall, and covering it by a protecting layer of plaster. Unfortunately, as a consequence of the plaster and thermal insulation layers mechanically acting as a mass-spring system, the accompanying mechanical resonance typically leads to a dip in the sound insulation spectrum in the low frequency range (usually $<500 \mathrm{~Hz}$ ), which, in the presence of outdoor traffic noise, is worsening the indoor acoustic comfort.

While the ISO 12354-1 [1] standard mentions the negative effect of ETICS resonances, especially when the massspring-mass resonance frequency lies above $200 \mathrm{~Hz}$ (see
Table D.3 of ISO 12354-1), scientific papers addressing this issue are scarse. Miskinis et al. [2] reported that a thermal layer made from expanded polystyrene (EPS), not covered by a plaster layer, does not influence the acoustic performance of a wall. The authors also mentioned that a finishing plaster layer is required to protect the EPS, and that it is providing additional sound insulation in the higher frequency range (i.e. above the ETICS resonance frequency). Although they reported the mass-spring-mass resonance frequencies for the tested ETICS walls, they did not quantify the sound reduction index at these resonance frequencies. Santoni et al. [3] developed a numerical model for the sound reduction index that takes into account the mechanical connections (dowels) that fix the thermal insulation of ETICS systems to the bare wall. However, they did not discuss the deterioration of the acoustic performance in the lower frequency range. In earlier publications, the present authors addressed the deterioration of the sound reduction index $R$ at the mass-spring-mass resonance of the ETICS system using numerical models $[4,5]$. Experimental evidence of the occurrence of the dip in the sound reduction index was given in [6]. The present work addresses the use of an array of dynamic vibration 
absorbers, sometimes also referred to as a locally resonant metamaterial structure, to counteract ETICS resonance induced dip in the noise reduction index.

A dynamic vibration absorber, DVA in short, basically consists of a seismic mass suspended on a spring - damper system. The same kind of device is sometimes also referred to as a tuned mass damper (TMD) [7]. The difference between a DVA and a TMD lies in the incentive for their use. A TMD is used to reduce the amplitude of the harmonic response of mechanical systems subject to tonal excitations. A DVA is typically used for wideband damping or for counteracting a large number of resonances in a wide frequency range. For a TMD, the natural frequency of the device is tuned to the tone of the excitation and its internal damping is set to be as low as possible. In case of a DVA, the resonance frequency of the device is tuned to a frequency in the range of modal frequencies that need to be suppressed and its internal damping is chosen to minimise the broadband resonant response of the controlled $\operatorname{mode}(\mathrm{s})$ [7].

Application of TMDs has shown to be very successful in noise mitigation strategies of propeller driven aircrafts where the fuselage is excited by strong aeroacoustic sound fields that are radiated by the propellers, causing high interior sound pressure levels. The generated sound spectrum consists of pure tones at the blade passing frequency and its harmonics. Fokker Aircraft [8] reported TMD induced sound reductions of the interior noise levels in the order of 8 to $10 \mathrm{~dB}$ at the fundamental blade passing frequency of $102 \mathrm{~Hz}$. This went at the cost of (about 25kg) extra weight, which is limited though, as the mass increase is reasonably small with respect to the total mass of the aircraft and the huge amount of absorbing material otherwise needed to obtain these reductions. The steady (controlled) rotational speed of the propeller, and thus fixed blade passing frequency, makes the use of TMDs the most appropriate choice in this specific case, giving large noise reductions at rather low frequencies. These reductions can also be achieved at take-off and landing conditions, by keeping the rotational speed of the propeller constant under all circumstances (and controlling the thrust by varying the propeller blade pitch when needed).

In case of broadband noise issues, DVAs are more suitable. Droz et al. [9] used DVAs to improve the sound reduction index of an aircraft fuselage panel. Experimental tests using a diffuse acoustic field showed that by using DVA resonators a reduction of up to $10 \mathrm{~dB}$ could be achieved at and around the ring mode frequency at the cost of about $5 \%$ mass increase of the fuselage panel. In another application, a DVA array with a subwavelength spacing was used to reduce the noise radiation of a car dash panel structure [10]. Different types of DVAs were used, tuned at three different frequencies, in an effort to obtain an improvement over a broader frequency range. Reductions of 5 - $13 \mathrm{~dB}$ were obtained.

Since façades are exposed to broadband acoustic excitation, and since the insulation dip in ETICS systems is pretty broad, DVAs are most appropriate. The question remains at which resonance frequency the DVAs should be tuned and how much internal damping gives the best result. These basic questions were addressed in the classical work of Ormondroyd and Den Hartog [11]. In this work, optimum values to minimise the displacement response of the host structure were given for the natural resonance frequency of DVAs and TMDs and their damping ratio. The natural resonance frequency $f_{D V A}$ of the DVAs (as well as TMDs) should be tuned according to the expression $f_{D V A}=f_{0} /(1+\mu)$, where $f_{0}$ is the resonant frequency of the host structure to be damped and $\mu$ is the ratio of the seismic mass of the DVA to the (modal) mass of the host structure. The optimal damping of the DVA, according to Ormondroyd and Den Hartog, is $\eta_{D V A}=\sqrt{3 \mu /\left(4(1+\mu)^{3}\right)}$, where $\eta_{D V A}$ is the structural loss factor of the DVA resonator. For more recent publications about the classic tuning theory of Ormondroyd and Den Hartog, see Thompson [12, 13]. These well established tuning laws can be straightforwardly employed to control the response at low resonance frequencies, provided that the modal overlap is not greater than unity [7].

In a numerical study, Van Belle [14] investigated the impact of internal structural damping of a DVA on the sound reduction index of infinite as well as finite plates. The work confirmed expectations that increasing damping broadens the frequency range where the DVA influences the sound reduction index, but reduces the peak of the sound reduction index improvement that can be obtained by DVAs.

De Melo Filho et al. [15] developed a thermoformed panel that incorporates DVAs to increase the sound reduction index in a specific frequency band. The low cost thermoforming production process is widely used, bringing resonant metamaterials closer to industrial applications.

A number of works focused on the improvement of the acoustic performance of panels or building partitions. For instance, Milica et al. [16] improved the sound reduction index of a single particle board by means of a periodical array of DVAs to counteract the coincidence dip in the insulation spectrum. Similarly, in a numerical study Liu et al. [17] used DVAs to improve the noise insulation of a sandwich plate around the coincidence region.

This paper focuses on the use of DVAs to counteract the mass-spring-mass resonance dip of ETICS systems. To the knowledge of the authors, no works addressing this specific application of DVAs have been published till now. In the present article, the effect of DVAs on the sound reduction index of ETICS walls is demonstrated numerically and experimentally. The numerical simulations combine a wavenumber domain approach with unit cell FEM-modelling employing Bloch-Floquet boundary conditions. The combined use of a Bloch-Floquet model with a wavenumber domain approach to compute the transmission loss of a wall, is new to the knowledge of the authors as well.

The paper is organized as follows. Section 2 describes 
acoustic measurements carried out in a transmission loss facility on a $10 \mathrm{~m}^{2}$ ETICS wall to reveal its behaviour and describe the 'ETICS dip' effect. In section 3 the wavenumber Fourier approach that is combined with unit cell FEMmodelling, using Bloch-Floquet boundary conditions, is presented. The method is applied to an ETICS wall in order to obtain a better understanding of the physical phenomena involved. The design of the DVAs is presented in Section 4. Section 5 reports on measurement results of the ETICS wall without and with DVAs. The results were obtained by laser Doppler vibrometry and by microphone based acoustic insulation measurements according to ISO 10140-2 [18]. Conclusions are presented in Section 6.

\section{Acoustic characteristics of ETICS walls}

The sound reduction index of a silicate cement brick wall with and without ETICS cladding was measured in the transmission loss facility of the KU Leuven acoustics laboratory according to the ISO 10140-2:2010 [18] standard. The volume of both source and receiving rooms was approximately $87 \mathrm{~m}^{3}$. The dimensions of the test opening were $3.30 \times 3.00 \mathrm{~m}^{2}$.

Two loudspeakers were used simultaneously in the source room, facing the two corners opposite to the test opening. Pink noise ranging from $63 \mathrm{~Hz}$ up to $5 \mathrm{kHz}$ was adopted, with a total sound pressure level of $108 \mathrm{~dB}$ ref. $20 \mu P a$.

The sound reduction index was measured twice, switching source and receiving rooms. According to reciprocity of acoustic wave propagation, the two sound reduction indices should be the same. However, small deviations can occur due to the non-diffusiveness of the acoustic fields, to the uncertainty in the measurement of the sound pressure levels in the source and in the receiving rooms and to the uncertainty of the reverberation time measurements in the receiving room caused by the limited number of measurement points. A numerical average of the two sound reduction index measurements was therefore taken.

The wall under test consisted of the following layers, see Fig. 1, mid graph, from left to right:

- gypsum plaster, thickness $3 \mathrm{~mm}$, density $750 \mathrm{~kg} / \mathrm{m}^{3}$;

- silicate cement brick wall, brick dimensions 300x200x200 mm, thickness $200 \mathrm{~mm}$, weight per brick: $22.23 \mathrm{~kg}$, density: $1855 \mathrm{~kg} / \mathrm{m}^{3}$;

- gypsum plaster, thickness $3 \mathrm{~mm}$, density $750 \mathrm{~kg} / \mathrm{m}^{3}$;

- Expanded polystyrene (EPS) thermal insulation, density $17 \mathrm{~kg} / \mathrm{m}^{3}$, thickness $140 \mathrm{~mm}$;

- reinforcement adhesive mortar based on lime and cement, thickness $7 \mathrm{~mm}$, density $1450 \mathrm{~kg} / \mathrm{m}^{3}$, with integrated reinforcement.

Details of the silicate cement brick wall being assembled in the opening separating the two rooms are shown in Fig. 1. The silicate cement brick wall was resting on the walls

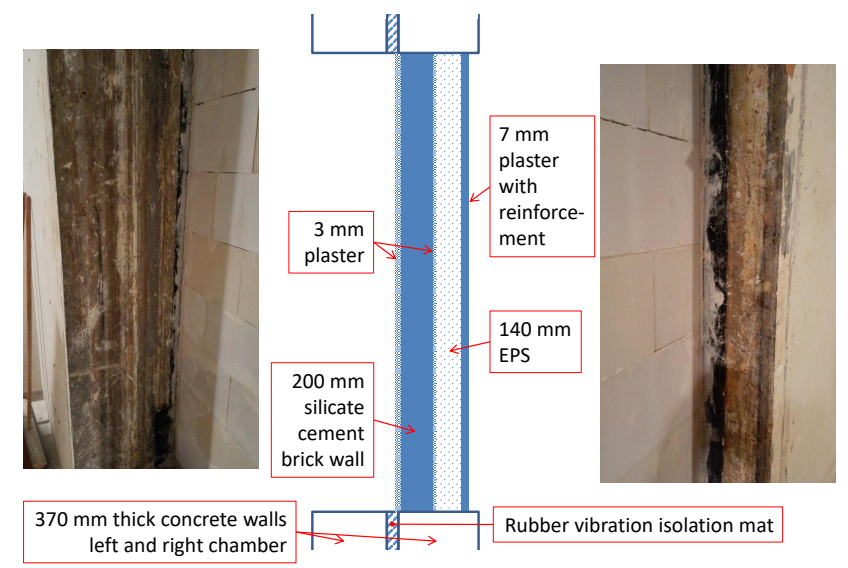

Figure 1: Construction of the ETICS wall in the test opening of the measurement facility at KU Leuven. Photos were taken after the silicate cement brick wall was build (plaster and EPS not applied yet).

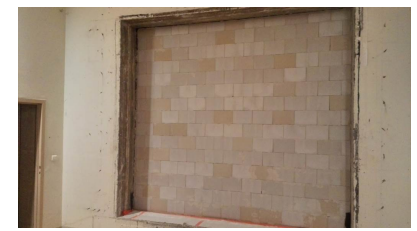

(a) Wall without plaster

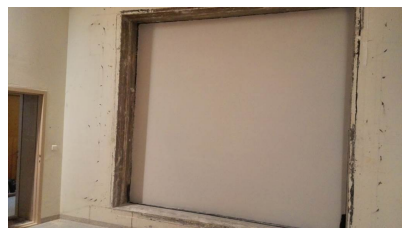

(b) Wall with plaster
Figure 2: Silicate cement brick wall with and without plaster.

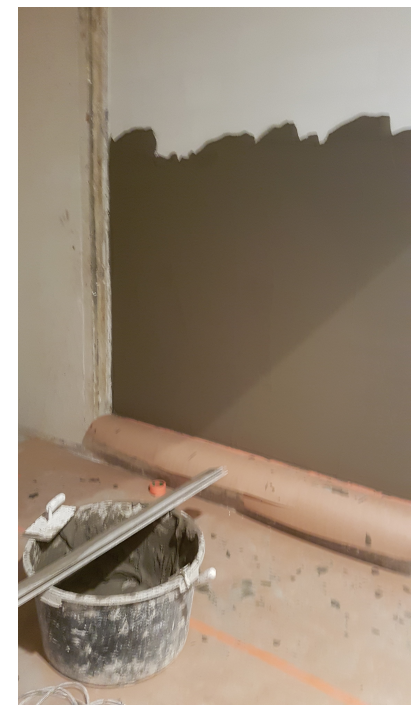

(a) Application of glue to the plastered silicate cement brick wall

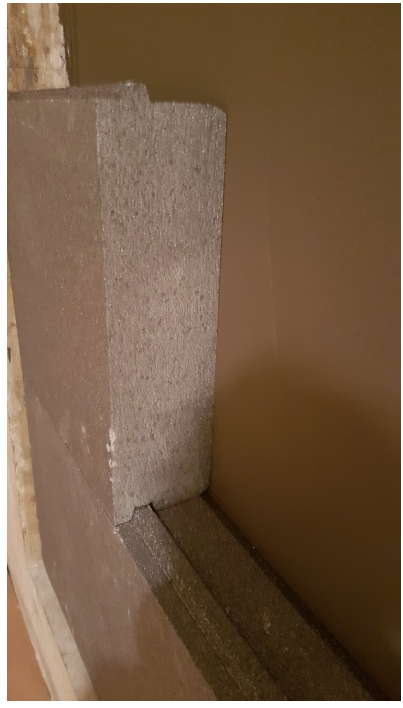

(b) Application of the EPS blocks to the glue
Figure 3: Gluing the EPS blocks to the silicate cement brick wall.

of one of the rooms only. The rubber vibration isolating mat between the source and receiving rooms, which was intended to minimize flanking transmission, was kept free.

A picture of the erected silicate cement brick wall is 
shown in Fig. 2a. Figure 2b shows the wall finished with plaster.

The EPS thermal insulation blocks were glued to the silicate cement brick wall, spreading glue across the entire surface (see Fig. 3). In this way, there was no need to make use of dowels to mechanically connect the EPS layer to the silicate cement bricks.

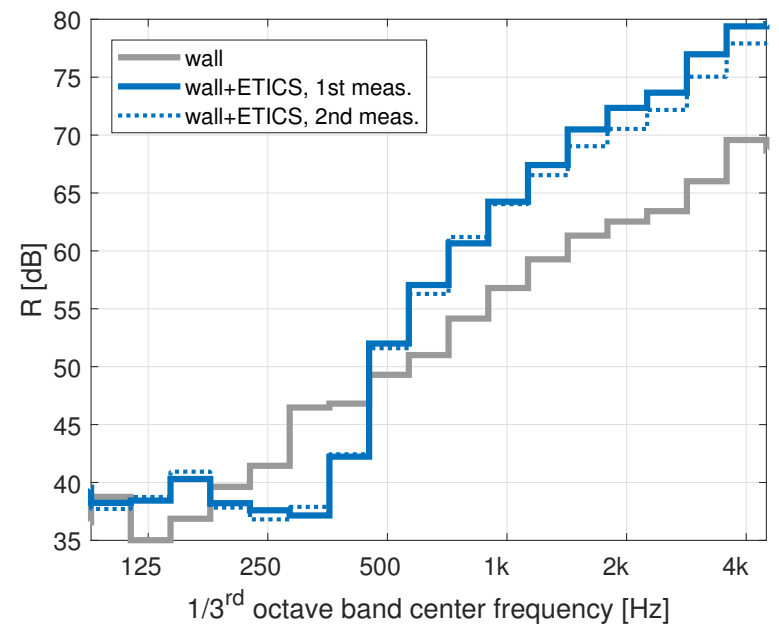

(a)

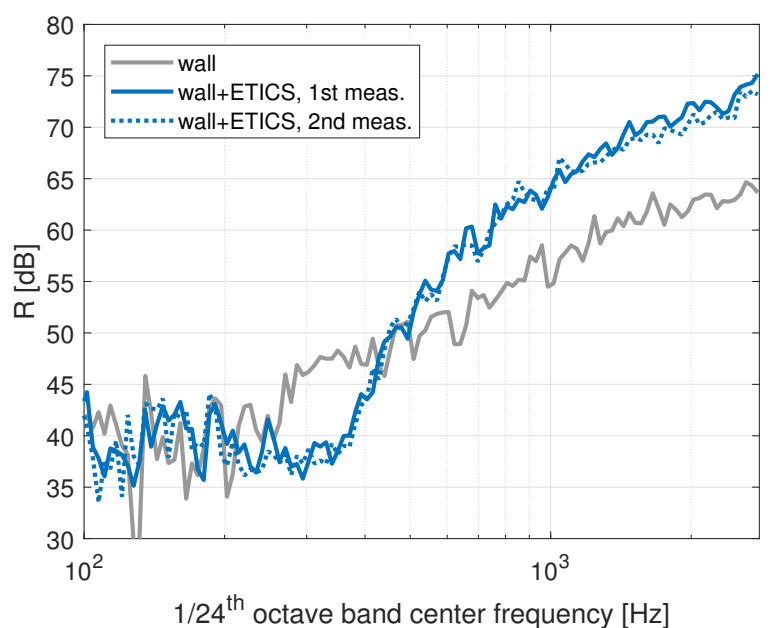

(b)

Figure 4: Sound reduction index $R$ of the bare wall and the ETICS wall, in (a) $1 / 3^{\text {rd }}$ octaves, and in (b) $1 / 24^{\text {th }}$ octaves.

The measured sound reduction spectra, obtained according to the ISO 10140-2 standard, on (i) the bare wall (gray solid line), (ii) the ETICS wall two weeks after the installation of the EPS+mortar coatings (blue solid line) and (iii) the ETICS wall 2 months after the installation of the EPS+mortar coatings (blue dashed line), are shown in Fig. 4, in $1 / 3$ (a) and $1 / 24$ octave bands (b). Below $200 \mathrm{~Hz}$, the trend of the sound reduction index for the bare wall is relatively flat. Above this frequency the curves follow a slope of $6 \mathrm{~dB} /$ octave. The spectra for the ETICS configurations follow roughly the same trend, but in addition they exhibit a mass-spring-mass resonance dip around
$320 \mathrm{~Hz}$. The loss of the sound reduction index in the $1 / 3$ octave band at $315 \mathrm{~Hz}$ is about $10 \mathrm{~dB}$. The frequency region were the ETICS wall influences the behaviour of the bare wall ranges between $250 \mathrm{~Hz}$ and $400 \mathrm{~Hz}$. In order to mitigate this ETICS induced loss of performance, two types of DVA were designed and tested. This aspect is discussed in the following sections.

\section{Theoretical modelling}

\subsection{ETICS walls}

In order to obtain a better understanding of the physics involved in the ETICS induced acoustic isolation dip, numerical models of the ETICS wall were built. Section 3.1.1 discusses the modelling of the structural dynamic response to airborne sound of the wall featuring an ETICS system. Section 3.1.2 discusses the modelling of the acoustic radiation of the vibrating wall by means of a Rayleigh integral in the wavenumber domain.

\subsubsection{Structural response}

The structural dynamics of the ETICS wall was modelled by means of a finite element model with BlochFloquet boundary conditions.

One of the first applications of Bloch-Floquet theory [19] in the finite element method was reported in 1995 [20]. Nowadays, the method is widely used in the analysis of periodic structures to increase computational efficiency without compromising accuracy. It should however be noted that the model assumes the structure to be spatially periodic, and thus of infinite lateral dimensions. This assumption limits the predictive capacity of the model for real life structures having finite dimensions. Nevertheless, the study of the wave propagation characteristics for an infinite wall can give valuable information.

Bloch-Floquet's theorem [19, 21] states that the propagation of a wave from cell to cell does not depend on the cell location within the periodic structure. Following Hussein [22], the displacements of adjacent Representative Elementary Volumes (REVs) of the periodic structure can be written as

$$
\mathbf{u}(\mathbf{r}+\mathbf{d}, \mathbf{k})=\mathbf{u}(\mathbf{r}, \mathbf{k}) e^{i\left(\mathbf{k}^{T} \mathbf{d}\right)}
$$

where $\mathbf{r}=\{x, y, z\}$ is the position vector, $\mathbf{u}=\left\{u_{x}, u_{y}, u_{z}\right\}$ is the displacement vector, $\mathbf{d}=\left\{d_{x}, d_{y}, d_{z}\right\}$ is the lattice spatial periodicity vector, $\mathbf{k}=\left\{k_{x}, k_{y}, k_{z}\right\}$ is the wave vector, and $i=\sqrt{-1}$. The real part of the wave vector $\mathbf{k}$ is inversely proportional to the phase velocity, while the imaginary part reflects the attenuation of the wave. By virtue of Bloch-Floquet's theorem, wave propagation through the entire periodic structure can be described by considering a single REV, with obvious reductions of the computational effort. 


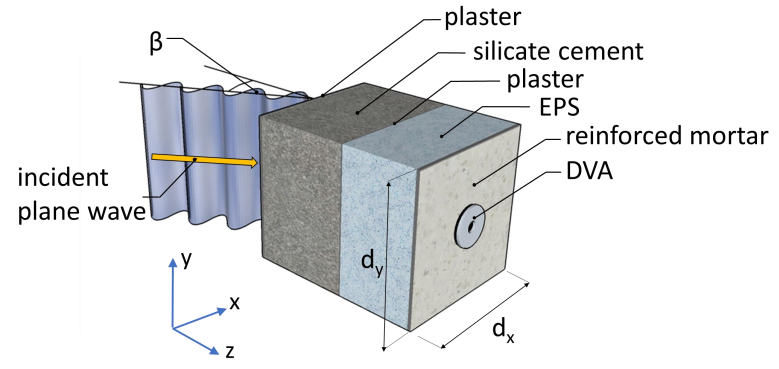

Figure 5: Representative Elementary Volume, REV, of the ETICS wall with DVAs.

\begin{tabular}{|l|l|l|l|l|}
\hline & $\begin{array}{l}\text { Young's } \\
\text { modulus } \\
{[\mathrm{GPa}]}\end{array}$ & density & $\begin{array}{l}\text { loss } \\
\text { factor } \\
{\left[\mathrm{kg} / \mathrm{m}^{3}\right]}\end{array}$ & $\begin{array}{l}\text { tick- } \\
\text { ness } \\
{[\mathrm{m}]}\end{array}$ \\
\hline Silicate cement & 12.3 & 1855 & 0.05 & 0.2024 \\
Expanded Polystyrene & $3.6 \mathrm{e}-3$ & 14 & 0.15 & 0.160 \\
Reinforced adh. mortar & 10 & 1450 & 0.08 & 0.007 \\
\hline
\end{tabular}

Table 1: Material properties and thicknesses used in the finite element model.

As the ETICS wall is a relatively thin-walled structure compared to the structural wavelength at the frequencies of interest ${ }^{1}$, only waves propagating in the lateral $x$ - and $y$ - directions need to be considered. For the sake of simplicity, in the present work the waves are assumed propagating in the lateral $x$-direction only. The geometry of one REV is presented in Fig. 5, showing the layered structure, typical of an ETICS wall. The spatial periodicity vectors $d_{x}$ and $d_{y}$ have a length (i.e. the dimension of the REV in those directions) of $0.31 \mathrm{~m}$ and $0.28 \mathrm{~m}$, respectively. The silicate cement wall $(200 \mathrm{~mm}$, $1855 \mathrm{~kg} / \mathrm{m}^{3}$ ) and the two plaster layers (each $3 \mathrm{~mm}, 750$ $\mathrm{kg} / \mathrm{m}^{3}$ ) on each side of the wall are modelled as a single layer with a density of $1855 \mathrm{~kg} / \mathrm{m}^{3}$ and a thickness of $(200 \times 1855+2 \times 3 \times 750) / 1855=202.4 \mathrm{~mm}$, thus yielding the same mass per unit area. The materials properties and thicknesses of the ETICS wall components used in the model are listed in Table 1. The material properties were taken from [24] and from the manufacturer of the silicate cement blocks. Poisson's ratio was assumed to be 0.33 for all materials mentioned in the table. The material properties and dimensions used in the model are representative of the configuration tested and are described in Section 5. The finishing plaster layer that protects the EPS is a

\footnotetext{
${ }^{1}$ Using the classical Love-Kirchhoff thin shell theory, the propagation speed for bending waves $c_{B}=\sqrt{1.8 c_{L} h f}$, where $c_{L}=$ $\sqrt{E / \rho\left(1-\nu^{2}\right)}$ is the longitudinal propagation speed, $f$ is the frequency, and $h$ is the thickness of the shell. The factor 1.8 is an approximate number for $2 \pi / \sqrt{12}$. For a wall with the properties listed in Table 1 , the wavelength to thickness ratio, $f / h c_{B}$, is larger than 7 for frequencies below $500 \mathrm{~Hz}$, which implies that the thin shell theory is, by approximation, sufficient to account for waves running in $x$-direction for frequencies below $500 \mathrm{~Hz}$. See [23] for more detailed considerations on the validity of the Love-Kirchhoff thin shell theory.
}

reinforced type of adhesive mortar, and will be called like that hereafter.

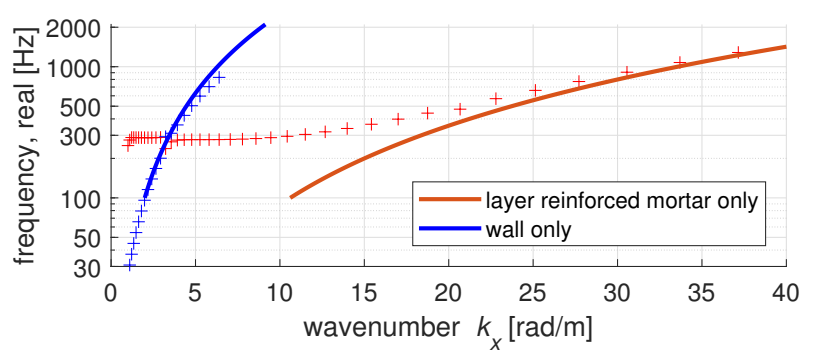

(a) Wavenumber dependence of the real part of the frequency

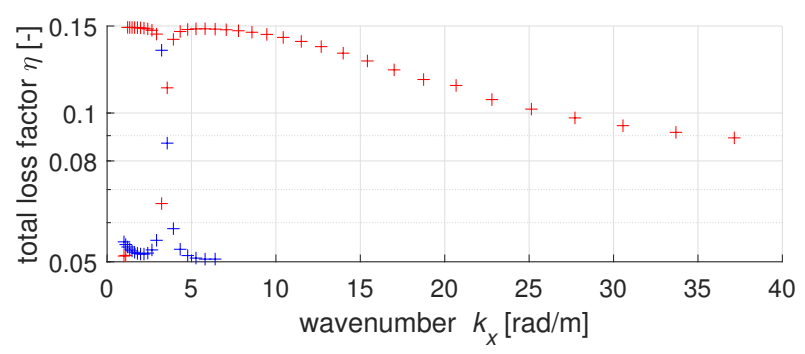

(b) Wavenumber dependence of the total loss factor $\eta$

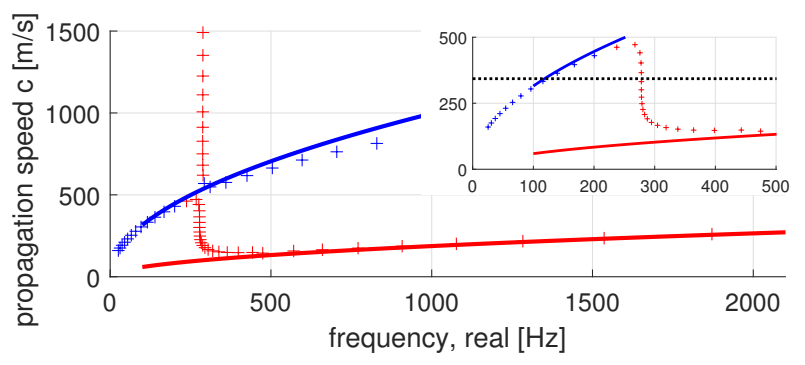

(c) Frequency dependence of the phase velocity

Figure 6: Dispersion curves of the ETICS wall. Crosses (blue and red): Bloch-Floquet model of ETICS wall. Solid curves: analytical dispersion curves for the silicate cement wall only (blue solid curve) and for the layer of reinforced adhesive mortar only (red solid curve) obtained with Love-Kirchhoff's thin plate theory.

Solving the eigenvalue problem for varying prescribed wavenumbers $k_{x}$, enforced as Bloch-Floquet boundary condition, the dispersion curves can be derived [25]. In the solution of the eigenvalue problem, the real part of the scanned wavenumber $k_{x}$ was prescribed and the resulting eigenfrequencies were allowed to be complex to include damped modes in the analysis. The results of the BlochFloquet computations are shown in Fig. 6. This figure also shows the Love-Kirchhoff's thin plate theory results for the silicate cement wall only and the layer of reinforced adhesive mortar only cases.

Two asymptotic waves occur for the lower and higher wavenumbers, i.e. the waves of the ETICS system that are dominated by the dynamics of the silicate cement wall (blue solid curve) and the layer of reinforced adhesive mortar (red solid curve) respectively.

Figure 6a shows that the group velocity $c_{g}=\partial \omega / \partial k$ approaches zero for the resonances close to $320 \mathrm{~Hz}$. This 
corresponds to the mass-spring-mass resonance frequency of the ETICS wall, i.e. the mode where the layer of reinforced adhesive mortar and the silicate cement wall act as masses, and the EPS layer acts as a spring.

The graph with the total loss factor ${ }^{2} \eta$ of the computed eigenmodes as function of wavenumber $k_{x}$ (Fig. 6b) shows that the modes that are dominated by the silicate cement wall (blue crosses) have a loss factor $\eta$ of about 0.05 , corresponding to the silicate cement loss factor given in Table 1. The modes at higher frequencies, which are dominated by the layer of reinforced adhesive mortar (red crosses), converge to a loss factor $\eta$ of about 0.08 , which corresponds to the plaster loss factor. Interestingly, for the modes at frequencies around $320 \mathrm{~Hz}$, the mass-spring-mass eigenfrequency (red crosses) exhibits a loss factor of about 0.15 , corresponding to the one assumed for EPS. This behaviour can be explained by the large deformations in the EPS layer for this eigenmode.

Finally, Fig. 6c shows the frequency dependence of the phase velocity of the bending waves, $c=\Re(\omega) / k$. The intersection (see inset figure) of the dispersion curve with the speed of sound in air, $343 \mathrm{~m} / \mathrm{s}$, corresponding to the lowest coincidence frequency $f_{c}$ (also known as the critical frequency), occurs at approximately $f_{c}=115 \mathrm{~Hz}$, well below the mass-spring-mass resonance frequency.

The numerically predicted coincidence frequency for the ETICS wall assembly is in good agreement with the analytically computed coincidence frequency for the bare silicate cement wall, which equals [32] $f_{c}=\frac{c_{0}^{2}}{2 \pi} \sqrt{\frac{\rho_{s} h}{E h^{3} /\left(12\left(1-\nu^{2}\right)\right)}} \approx$ $115 \mathrm{~Hz}$, using $c_{0}=343 \mathrm{~m} / \mathrm{s}$ and the material properties of the silicate cement wall as listed in Table 1. From this result it can be concluded that the coincidence effect of the silicate cement wall with ETICS is dominated by the dynamic behaviour of the silicate cement wall.

The measurement results in Fig. 4a show a minimum of the sound insulation index $R$ of the bare wall in the 125 $\mathrm{Hz} 1 / 3^{r d}$ octave frequency band, which is in agreement with the above identified coincidence frequency. The wall with ETICS shows a minimum plateau in the 100 and $125 \mathrm{~Hz} 1 / 3^{r d}$ octave frequency bands. However, the measurement data are not conclusive at this point. The large measurement uncertainty is due to the non-diffusiveness of the sound fields in sending and receiving rooms, with commonly known difficulties to determine the sound insulation index $R$ at low frequencies [27, 28]

Using the finite element model with Bloch-Floquet boundary conditions, the structural response of the ETICS wall assembly to a plane incident airborne sound wave was calculated for different frequencies and angles of incidence.

Figure 7 shows the magnitude of the velocity response of the wall to an acoustic plane wave impinging the wall as a function of frequency and angle of incidence $\beta$. Here $\beta=0$ radians corresponds to the normal incidence case

\footnotetext{
${ }^{2}$ Note that the loss factor $\eta=\Im\left(\omega^{2}\right) / \Re\left(\omega^{2}\right) \approx 2^{\Im}(\omega) / \Re(\omega)$ for small values of $\eta[26]$, where $\omega$ is the complex eigenvalue.
}

and $\beta=\pi / 2$ radians corresponds to the grazing incidence case (see Fig. 5). While varying the angle of incidence, in accordance to the boundary conditions, the structural wavenumber was forced to be equal to the projected acoustic wavenumber of the exciting field, also called the trace wavenumber:

$$
k_{x}=k_{0} \sin (\beta)=\frac{\omega \sin (\beta)}{c_{0}}
$$

where $k_{0}$ is the acoustic wavenumber in air, and $c_{0}$ is the speed of sound in air $(343 \mathrm{~m} / \mathrm{s})$.

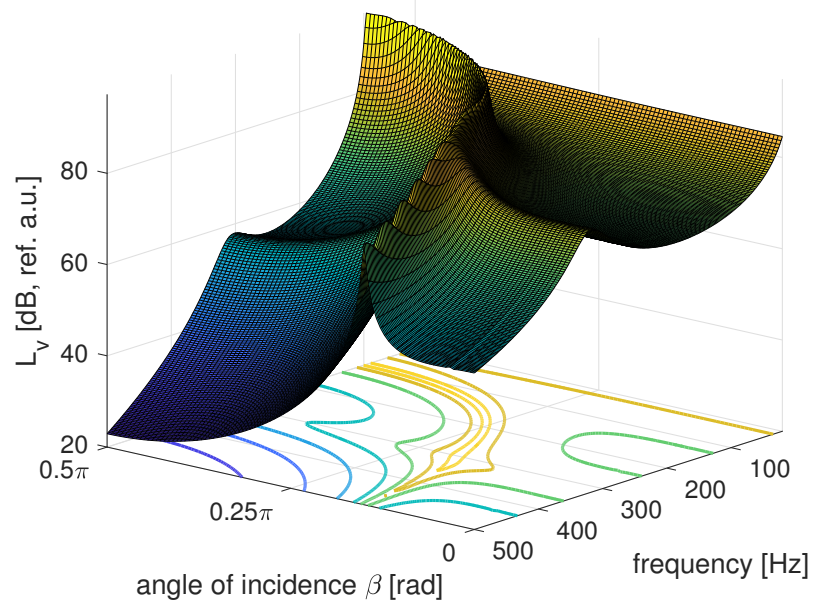

Figure 7: Numerically predicted velocity response of the ETICS wall due to acoustic excitation as a function of frequency and angle of incidence $\beta$.

For $\beta=\pi / 2$ radians, a peak in the response of the wall occurs at the critical frequency $\left(f_{c}=115 \mathrm{~Hz}\right)$. For lower angles of incidence, the coincidence frequency shifts to higher values. For instance, at $\beta=0.6458 \mathrm{rad}$ (37 deg) a peak in the response occurs at $280 \mathrm{~Hz}$, for which the projected wavenumber or trace wavenumber equals $k_{x}=$ $3.08 \mathrm{rad} / \mathrm{m}, c_{x}=2 \pi f / k_{x}=571 \mathrm{~m} / \mathrm{s}$. All coincidence related peaks are caused by a resonance of the wall with ETICS, dominated by the silicate cement wall motion (blue crosses in Fig. 6a,6c).

Around a frequency slightly above $320 \mathrm{~Hz}$, corresponding to the mass-spring-mass ETICS resonance, an increase in the velocity response occurs for all the angles of incidence (see Fig. 7). This increase is smaller than the response at the silicate cement wall-dominated eigenmodes, as the model assumes that the mass-spring-mass resonance has a higher loss factor $(\eta=0.15)$ as compared to the one of the silicate cement wall-dominated modes $(\eta=0.05)$.

\subsubsection{Acoustic response computation in wavenumber do- main using Bloch-Floquet FEM model}

In this section, the wavenumber domain approach is combined with the unit cell FEM-modelling approach that uses Bloch-Floquet boundary conditions to compute the sound reduction index $R$ of the ETICS wall. The 
wavenumber Fourier approach was originally developed by Heckl in 1977 [29] and later on it has been widely used in acoustics [30, 31]. In applied mechanics the method is referred to as the integral transform method (ITM) [33].

For brevity, the dependence on the angular frequency $\omega$ is omitted hereafter. The velocity of the wall in wavenumber domain, $\dot{U}_{z}$, can be computed from

$$
\dot{U}_{z}\left(k_{x}, k_{y}\right)=\iint_{S} \dot{u}_{z}(x, y) e^{-i\left(k_{x} x+k_{y} y\right)} d x d y
$$

where $\dot{u}_{z}$ is the velocity of the wall measured in normal direction ( $z$-direction), $k_{x}$ and $k_{y}$ are the wavenumbers in $x$ - and $y$-directions, and $S$ is the surface of the baffled wall. From $\dot{U}_{z}$, the acoustic pressure $P$ in the wavenumber domain at the receiving side of the wall can be computed from [31]

$$
P\left(k_{x}, k_{y}\right)=\frac{\rho_{0} c_{0} k_{0}}{k_{z}} \dot{U}_{z}\left(k_{x}, k_{y}\right)
$$

where $\rho_{0}$ is the density of air and $k_{z}$ is the acoustic wavenumber in normal direction to the wall, with

$$
k_{z}=\sqrt{k_{0}^{2}-k_{x}^{2}-k_{y}^{2}}
$$

From the acoustic pressure and the acoustic particle velocity, which is equal to the structural velocity of the wall, the acoustic intensity and thus the radiated active sound power $\Pi$ can be calculated by the following Rayleigh integral solved in the wavenumber domain [31]

$$
\begin{aligned}
\Pi & =\frac{\rho_{0} c_{0} k_{0}}{8 \pi^{2}} \int_{S_{r}} \frac{\left|\dot{U}_{z}\left(k_{x}, k_{y}\right)\right|^{2}}{k_{z}} d k_{x} d k_{y} \\
& =\frac{\rho_{0} c_{0} k_{0}}{8 \pi^{2}} \int_{S_{r}} \frac{\left|\dot{U}_{z}\left(k_{x}, k_{y}\right)\right|^{2}}{\sqrt{k_{0}^{2}-k_{x}^{2}-k_{y}^{2}}} d k_{x} d k_{y}
\end{aligned}
$$

where $S_{r}$ is the area inside and including the radiation circle, defined as $k_{x}^{2}+k_{y}^{2}=k_{0}^{2}$.

In the Bloch-Floquet approach, the velocity field is calculated at a discrete number of spatial points and within the REV only. For the model used, the REV had a dimension of $\left|d_{x}\right|=0.31 \mathrm{~m}$ and $\left|d_{y}\right|=0.28 \mathrm{~m}$. The structural response was calculated for frequencies from $20 \mathrm{~Hz}$ to $500 \mathrm{~Hz}$ for all angles of incidence. The enforced (trace) structural wavenumbers $k_{x}$ range from 0 up to the wavenumber in air at $500 \mathrm{~Hz}$, which equals $k_{0}=2 \pi 500 / c_{0}=9.16 \mathrm{rad} / \mathrm{m}$. Thus, in this specific case, the trace wavenumber varied between 0 and $9.16 \mathrm{rad} / \mathrm{m}$, which corresponds to wavelengths ranging from infinity down to $\lambda=2 \pi / k_{0}=0.68$ m. These wavelengths, even the smallest ones, are (much) longer than the characteristic dimensions of the REV. For this reason the calculation of the structural velocity field in the (discrete) wavenumber domain based on the velocity computed in the REV domain only cannot be done accurately. The wavenumber resolution $\Delta k_{x}=2 \pi / d_{x}=$ $22 \mathrm{rad} / \mathrm{m}$ is rather poor, being larger than the maximum structural wavenumber $k_{x}$.
This problem can be resolved [25] by periodically replicating the discrete velocity field beyond the REV and exploiting Bloch-Floquet's theorem. Applying Eq. 1 in the $x$-direction, the replication process can be written as

$$
\dot{\mathbf{u}}\left(x+n d_{x}\right)=\dot{\mathbf{u}}_{R E V}(x) e^{i\left(n k_{x} d_{x}\right)}, n=1 \ldots N
$$

where $\dot{\mathbf{u}}_{R E V}$ is the velocity vector for the REV, and $N$ is an integer number that is large enough (typically) to obtain a representation of the waves allowing a reasonably accurate transformation to the wavenumber domain. In practice, for calculating the radiated sound power by means of the approach outlined above, only the velocity in the $z$-direction, $\dot{u}_{z}$, is required.

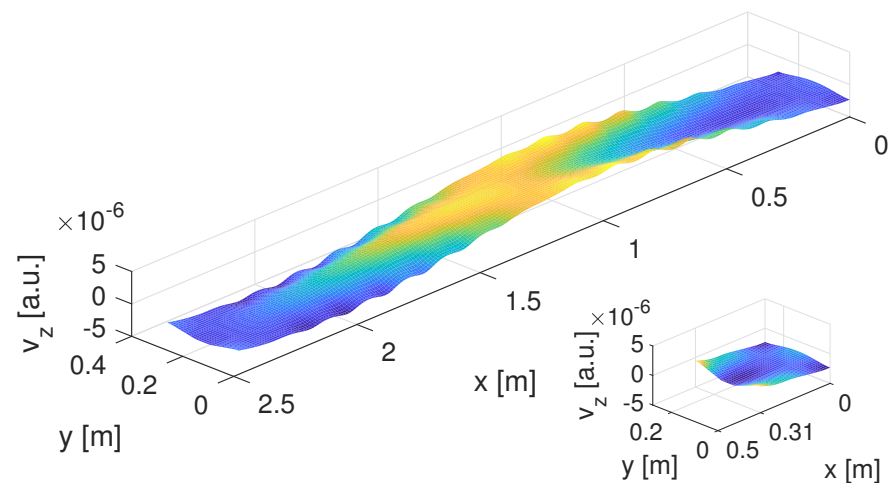

Figure 8: Numerically predicted normal component of the velocity field of the vibrating ETICS wall with DVAs, replicating the REV solution $N=8$ times in $x$-direction, for a frequency $f=300 \mathrm{~Hz}$, angle of incidence $\beta=30 / 180^{*} \pi \mathrm{rad}, k_{x}=2.75 \mathrm{rad} / \mathrm{m}$ and $\lambda_{x}=2.29 \mathrm{~m}$. The inset shows the velocity field within the REV only (dimension 0.31 $\mathrm{m} \times 0.28 \mathrm{~m})$.

To illustrate this procedure, Fig. 8 shows the velocity field of an ETICS wall featuring a set of dynamic vibration absorbers (DVAs). The particular effect of DVAs will be discussed in more detail in Section 3.2. The graph was obtained by using a replication factor $N=8$, thus expanding the solution in the spatial $x$-direction up to $8 \times$ $0.31 \mathrm{~m}=2.48 \mathrm{~m}$. Using this replication factor, about one wavelength can be observed for the frequency and angle of incidence considered ( $\lambda_{x}=2.29 \mathrm{~m}$ in this specific case), thus allowing to make a valid conversion to wavenumber domain by taking a spatial Fourier transform over the replicated domain. In order to allow for a robust Fourier transform over a wide range of frequencies and angles of incidence, a replication factor $N=256$ was used in subsequent calculations, thus expanding the spatial computational domain to a length of $256 \times 0.31 \mathrm{~m}=$ almost $80 \mathrm{~m}$. Using this replication factor, the wavenumber domain resolution $\Delta k_{x}$ equals $2 \pi / N d_{x}=0.08 \mathrm{rad} / \mathrm{m}$. In addition, a Hanning window in the $x$-direction was applied to reduce leakage.

An example of the wavenumber domain representation of the considered spatial field using $N=256$ is shown in Fig. 9 for $f=300 \mathrm{~Hz}$ and $\beta=30 \mathrm{deg}$ (corresponding with $\left.\lambda_{x}=2.29\right)$. The figure also shows the radiation circle $k_{x}^{2}+$ $k_{y}^{2}=k_{0}^{2}$. Note that wave components outside the radiation 


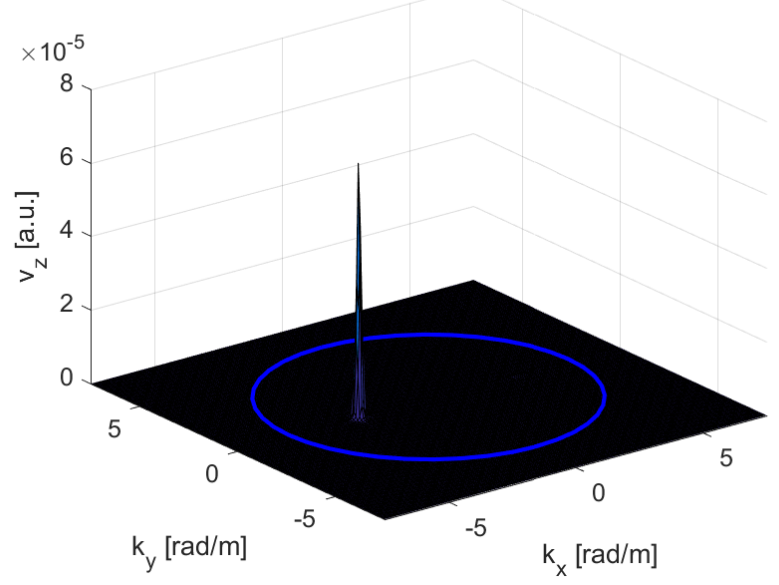

(a)

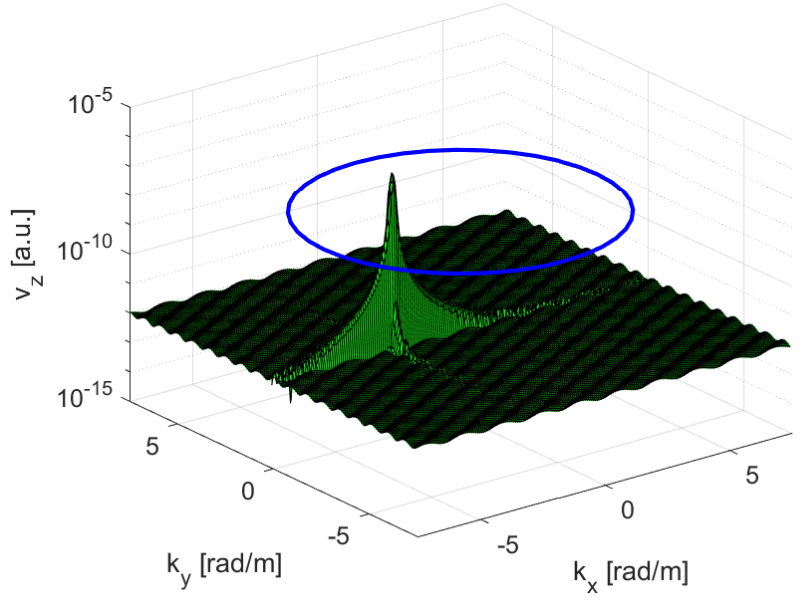

(b)

Figure 9: Spatial dependence of the numerically predicted normal velocity component of he vibrating wall, for a frequency $f=300 \mathrm{~Hz}$, an angle of incidence $\beta=30 / 180 \cdot \pi \mathrm{rad}, k_{z}=2.75 \mathrm{rad} / \mathrm{m}$ and $\lambda_{x}=2.29$ m. Spatial Fourier transform taken after replicating the REV solution 256 times along the $x$ - and $y$-directions. The radiation circle $k_{x}^{2}+k_{y}^{2}=k_{0}^{2}$ is indicated by a solid blue circle. (a) Linear scale; (b) Logarithmic scale.

circle are evanescent waves [30], which do not contribute to the active sound power, and thus do not need to be considered in the radiated sound power calculations (cfr Eq. 6). Also note that the side-skirts visible in Fig. 9b are caused by the wiggles at $y=0 \mathrm{~m}$ and at $y=0.18 \mathrm{~m}$ that can be noticed in Fig. 8. These anomalies are due to the presence of the DVAs that were used in this model, as will be discussed further in Section 3.2.

Whilst, for clarity, Fig. 9 shows a wavenumber spectrum for which the solution at REV-level was replicated in both $x$ and $y$-directions, in practice, replicating the wave field in $x$-direction only is sufficient. The reason is that only plane waves propagating in $x$-direction have been considered, using a Bloch-Floquet boundary condition. This implies that the Bloch-Floquet boundary condition in $y$-direction reads $\mathbf{u}(x, y+d, z)=\mathbf{u}(x, y, z)$, which means that the wavefield as shown in Fig. 8 is repeated indefinitely in the $y$-direction. Thus, taking the discrete Fourier transform in $y$-direction using the REV-solution only (without replicating in $y$ - direction), gives exact results in wavenumber domain for that part of the (2D) transform.

By means of Eq. 6, the radiated sound power can be computed from the velocity fields. The intensity of the acoustic field impinging the wall is given by

$$
p_{i} v_{n}=p_{i} v_{i} \cos (\beta)=\frac{p_{i}^{2} \cos (\beta)}{\rho_{0} c_{0}}=\frac{p_{b l}^{2} \cos (\beta)}{4 \rho_{0} c_{0}}
$$

where $p_{i}$ is the incident pressure, $p_{b l}$ is the blocked pressure $\left(p_{b l}=2 p_{i}\right), v_{n}$ is the acoustic particle velocity normal to the wall, and $v_{i}$ is the acoustic particle velocity along the wave vector of the incident wave. Thus, the sound reduction index $R$ of the ETICS wall can be computed as

$$
R=10 \log _{10}\left(\frac{p_{b l}^{2} S \cos (\beta)}{4 \rho_{0} c_{0} \Pi}\right)
$$

where $S$ is the surface area of the wall.

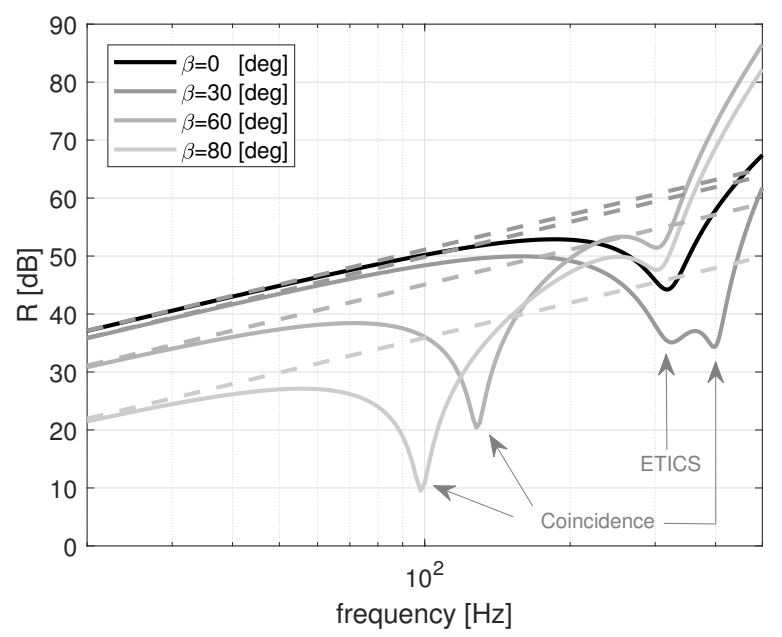

Figure 10: Numerically predicted sound reduction index $R$ (solid curves) and mass-law predicted sound reduction index (dashed curves). The ETICS dip is predicted at a frequency of approximately $320 \mathrm{~Hz}$.

The resulting sound reduction index is shown in Fig. 10 for a number of angles of incidence $\beta$. For comparison, the figure also shows the mass-law predicted sound reduction index $[32,34]$

$$
R_{\text {mass-law }}=10 \log _{10}\left[1+\left(\frac{\omega \rho_{s} \cos (\beta)}{2 \rho_{0} c_{0}}\right)^{2}\right]
$$

where $\rho_{s}$ is the mass per unit area of the wall. At low frequencies, well below the coincidence frequency, the curves coincide, providing a validation of the numerical approach 
for low frequencies. A number of dips in the sound reduction index $R$ of the ETICS wall can be observed. Two types of dip occur: one typically occurs due to the ETICS mass-spring-mass resonance (indicated by the arrow 'ETICS' in Fig. 10), which has a fixed frequency of about $320 \mathrm{~Hz}$. The other is related to the coincidence dip (indicated by the arrow 'Coincidence' in Fig. 10), and depends on the angle of incidence.

It should be remarked that, for the computation of the sound reduction index $R$ of a relatively simple ETICS wall without DVAs, it is not necessary to use the procedure of replicating the data and exploit the Fourier-domain approach as defined by equations 3 - 6 . In such a case the simple plane-wave model the problem reduces to the transmission of an obliquely incident sound field through an unbounded flexible partition, for which $R$ is given by [32]

$$
R=10 \log _{10}\left(\frac{p_{b l}^{2} \cos ^{2}(\beta)}{4 \rho_{0}^{2} c_{0}^{2}<\dot{u}_{z}^{2}>}\right)
$$

where $p_{b l}$ is the blocked surface pressure and $\dot{u}_{z}$ is the computed velocity in normal direction to the wall (e.g. as shown in Fig. 7) and $<>$ denotes spatially averaging. However, in the presence of DVAs the structural wavefields, and thus also the acoustic wavefields, become more complex (cfr. Fig. 8). To capture these effects the Fourierdomain approach is the appropriate method.

\subsection{ETICS walls with DVAs}

In this section, numerical results derived from an FEmodel of the ETICS wall including DVAs are presented. The same calculation approach as the one described in the earlier sections was used. The DVAs were modelled as damped spring-mass systems, where the spring and the seismic mass were made of solid elements, in contact with the reinforced adhesive mortar layer placed over an area of about $0.5 \mathrm{~m}^{2}$. The two important parameters characterising a DVA are the weight of its seismic mass and the resonance frequency. For the spring, we assumed a fixed structural loss factor $\eta=0.44$, corresponding with the mean damping factor of DVA Type 1 and Type 2. The choice of the elastic-damping material and the description of the two types of DVA will be discussed in Section 4.

An REV of the ETICS wall as illustrated in Fig. 5 was modelled, including the DVA that is mounted on the layer of reinforced adhesive mortar of the ETICS wall. BlochFloquet boundary conditions were applied, making the structure infinite in lateral directions as explained in Section 3.1.1. The DVAs were positioned at inter-distances indicated by $\left\{d_{x}, d_{y}\right\}$. The seismic mass of the DVA is denoted by $m_{D V A}$.

The classical tuning laws of Ormondroyd and Den Hartog can be straightforwardly employed to control the response at low resonance frequencies provided that the modal overlap is not greater than unity [7]. Considering the results obtained from the previous section (Fig.6 in particular) it can be concluded that these conditions are met. Referring to figs. 6, 7 and 10 it can be observed that the mass-spring-mass resonance of the ETICS system is situated around $320 \mathrm{~Hz}$. In the numerical model discussed in this section the DVAs were initially tuned at the ETICS mass-spring-mass resonance frequency $f_{D V A}=320 \mathrm{~Hz}$.

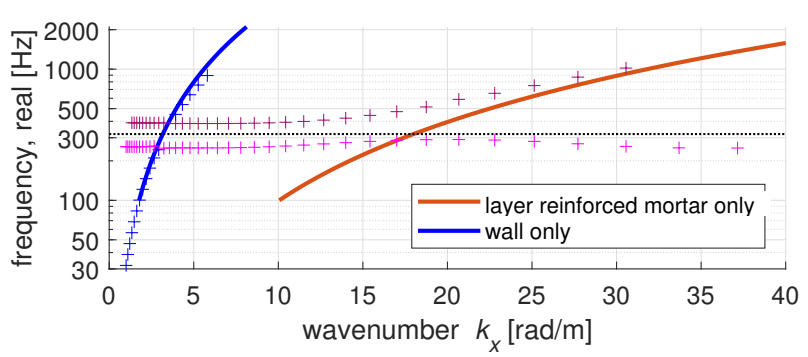

(a) Wavenumber dependence of the real part of the frequency

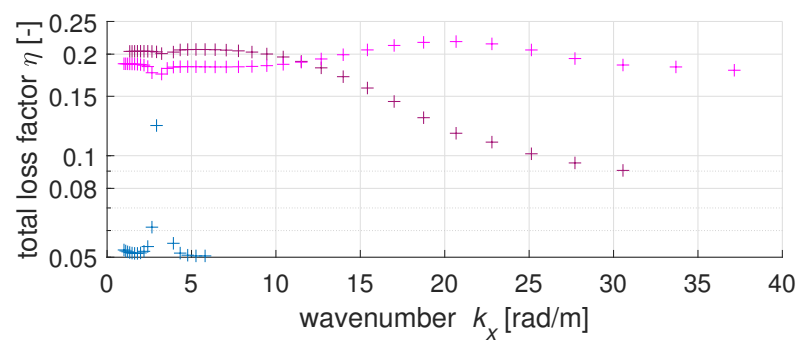

(b) Wavenumber dependence of the total loss factor $\eta$

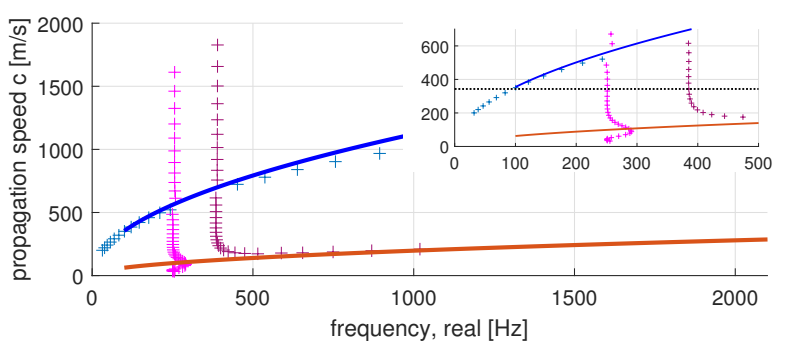

(c) Frequency dependence of the phase velocity

Figure 11: Dispersion curves ETICS wall with DVAs tuned at $f_{D V A}=320 \mathrm{~Hz}$, seismic mass $m_{D V A}=0.146 \mathrm{~kg}, \mu_{s c}=0.45 \%, \mu_{m l}$ $=17 \%$ (DVA resonance frequency of $320 \mathrm{~Hz}$ indicated by dashed lines in subfigs a and c), for a DVA inter-distance in the $x$-direction of $0.31 \mathrm{~m}$. Crosses (navy blue, cerise and purple): Bloch-Floquet model of ETICS wall. Solid curves: analytical dispersion curves for the silicate cement wall only (blue curve) and for a layer of reinforced adhesive mortar only (red curve), obtained by Love-Kirchhoff's thin plate theory.

In the theory of Omondroyd and Den Hartog, $\mu$ is defined as the ratio of the seismic mass of the DVA to the modal mass of the host structure. Here we introduce two more straightforward mass ratios that will be used throughout the paper. The mass increase due to the presence of the DVAs, relative to the mass of the silicate cement wall and relative to the reinforced adhesive mortar 
layer, denoted by $\mu_{s c}$ and $\mu_{m l}$, respectively, are defined as

$$
\begin{aligned}
\mu_{s c} & =\frac{m_{D V A}}{\rho_{s c} h_{s c} d_{x} d_{y}} \\
\mu_{m l} & =\frac{m_{D V A}}{\rho_{m l} h_{m l} d_{x} d_{y}}
\end{aligned}
$$

where $\rho_{s c}$ and $\rho_{m l}$ is the mass density of silicate cement and the mortar layer, respectively, and $h_{s c}$ and $h_{m l}$ is the thickness of the silicate cement wall and the mortar layer, respectively.

Solving the Bloch-Floquet eigenvalue problem for the ETICS wall with DVAs with a seismic mass $m_{D V A}$ of 0.146 $\mathrm{kg}$ and a DVA inter-distance of $\left\{d_{x}, d_{y}\right\}=\{0.31,0.28\} \mathrm{m}$ yields the results shown in Fig. 11. Using the material properties and thicknesses listed in Table 1, the relative mass increase amounts $\mu_{s c}=0.45 \%, \mu_{m l}=17 \%$, in this case. The modal mass $\mu$ is about $20 \%$. The results are compared with analytical solutions for (i) the silicate cement wall only and (ii) a layer of reinforced adhesive mortar (solid curves), both computed by means of Love-Kirchhoff's thin plate theory. Compared to the bare ETICS wall, which exhibits a single mass-spring-mass resonance (Fig. 6), in this case two resonances occur, one at $250 \mathrm{~Hz}$ and one at $385 \mathrm{~Hz}$. This reflects the presence of two eigenmodes, for which, respectively, the seismic mass of the DVA vibrates in-phase (the lower eigenfrequency) and oppositely (the higher eigenfrequency) with the ETICSsystem.

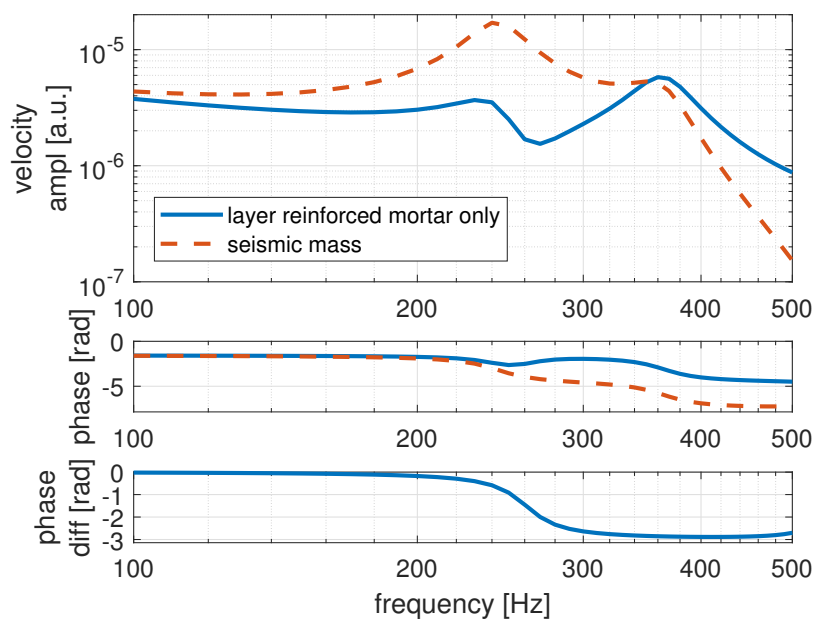

Figure 12: Numerically predicted frequency dependence of the velocity response of the ETICS wall with DVAs to perpendicularly incident $(\beta=0 \mathrm{rad})$ airborne sound, $\left\{d_{x}, d_{y}\right\}=\{0.31,0.28\} \mathrm{m}$, $f_{D V A}=320 \mathrm{~Hz}, m_{D V A}=0.146 \mathrm{~kg}, \mu_{s c}=0.45 \%, \mu_{m l}=17 \%$.

Figure 12 shows the spectrum of the structural response of the wall to perpendicularly incident $(\beta=0 \mathrm{rad})$ airborne sound, evaluated at a point of the plaster just in between two DVAs and the response of the seismic mass of the DVAs. The above mentioned resonances at $250 \mathrm{~Hz}$ and at $385 \mathrm{~Hz}$ are clearly visible, although, due to damping, the maximum amplitudes occur at slightly lower frequencies. A minimum in the response of the plaster occurs in between the two resonance frequencies. The plaster and seismic mass vibrate in phase at and below the first resonance frequency, and in counterphase at and above the second resonance frequency, confirming the phenomenology mentioned above. (a) $f=240 \mathrm{~Hz}$.

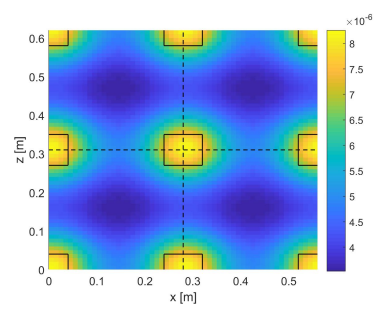

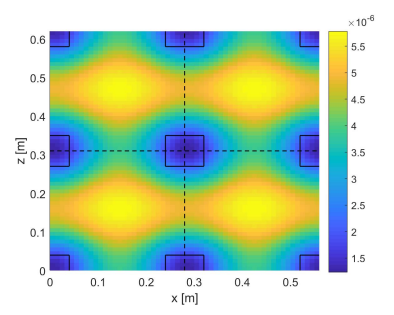

(b) $f=360 \mathrm{~Hz}$.
Figure 13: Numerically predicted spatial velocity field of the ETICS wall with DVAs, in response to perpendiculary incident ( $\beta=0 \mathrm{rad})$ airborne sound, $\left\{d_{x}, d_{y}\right\}=\{0.31,0.28\} \mathrm{m}, f_{D V A}=320 \mathrm{~Hz}$, $m_{D V A}=0.146 \mathrm{~kg}, \mu_{s c}=0.45 \%, \mu_{m l}=17 \%$. DVAs positions are indicated by black solid lines. Symmetry lines are indicated by black dashed lines.

The spatial vibration patterns, as computed by the Bloch-Floquet model, are shown in Fig. 13 for the two peak frequencies. The span-of-control of the DVAs, i.e. the spatial region for which a reduction in the vibration levels can be obtained, is limited, due to the finite (low) stiffness of the $7 \mathrm{~mm}$ layer of reinforced adhesive mortar. Interestingly, Fig. 12 shows that at $240 \mathrm{~Hz}$ the DVA is vibrating at a much higher level than the layer of reinforced adhesive mortar, 'pulling' the layer of adhesive mortar with in-phase-vibrations, whilst at $360 \mathrm{~Hz}$, the DVA is vibrating at about the same level as compared to the layer of reinforced adhesive mortar (out-of-phase).

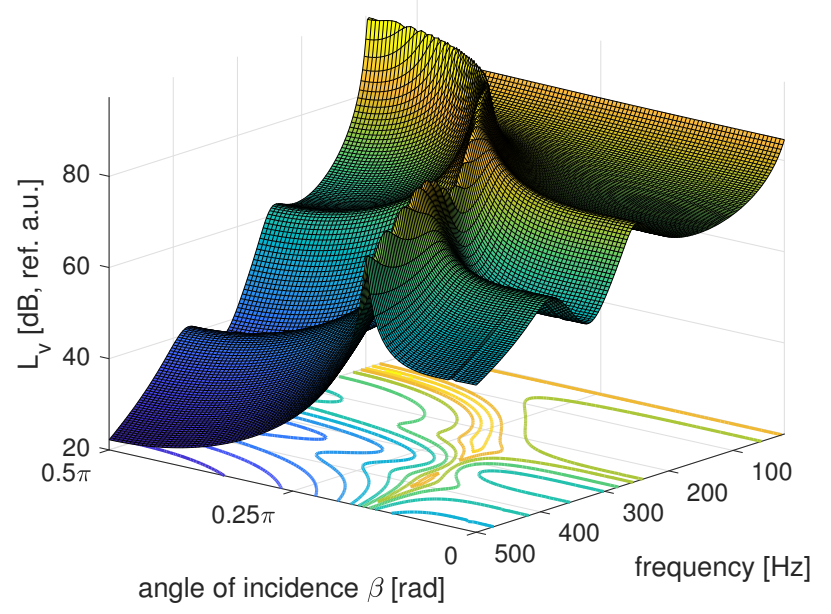

Figure 14: Numerically predicted velocity response of the ETICS wall with DVAs due to acoustic excitation as a function of frequency and angle of incidence $\beta,\left\{d_{x}, d_{y}\right\}=\{0.31,0.28\} \mathrm{m}, f_{D V A}=320 \mathrm{~Hz}$, $m_{D V A}=0.146 \mathrm{~kg}, \mu_{s c}=0.45 \%, \mu_{m l}=17 \%$.

Following the same approach as in the previous section, the structural response to an incident plane acoustic wave 
of the ETICS wall featuring the DVA was calculated. Figure 14 shows that the minimum in the response in between the two resonance frequencies, at $250 \mathrm{~Hz}$ and $385 \mathrm{~Hz}$, occurs for all angles of incidence.

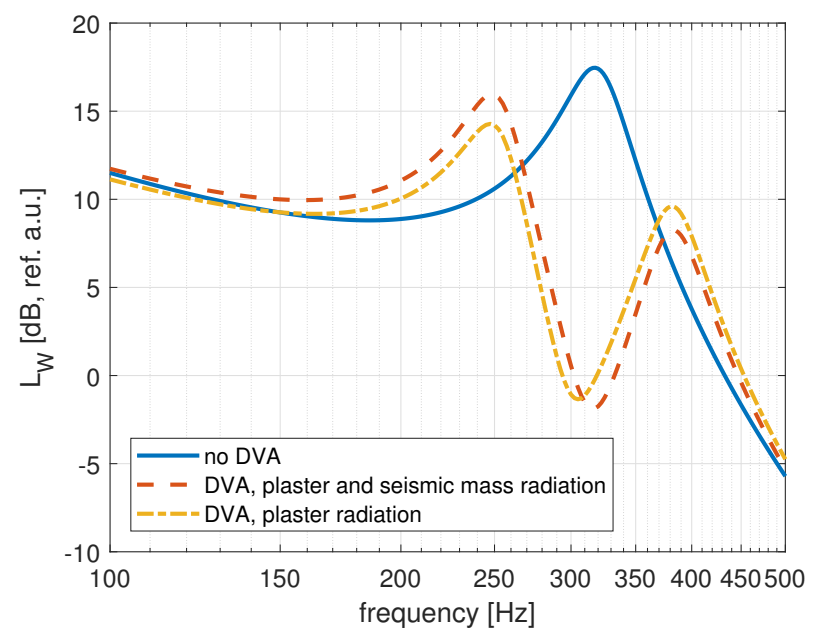

Figure 15: Numerically predicted sound power radiated by the ETICS wall wihout and with DVAs $\left(d_{x}=0.28 \mathrm{~m}, d_{y}=0.31 \mathrm{~m}\right.$, $\left.f_{D V A}=320 \mathrm{~Hz}, m_{D V A}=0.146 \mathrm{~kg}, \mu_{s c}=0.45 \%, \mu_{m l}=17 \%\right)$ due to normal incident $(\beta=0 \mathrm{rad})$ plane wave acoustic excitation as a function of frequency.

Using a wavenumber Fourier transform (section 3.1.2) the sound power radiated by the acoustically excited ETICS wall with DVAs was calculated, replicating the velocity field in the $x$-direction, using a replication factor $N=256$ and a Hanning window. As the ETICS-dip occurs at a fixed frequency, independently from the angle of incidence $\beta$ (see Fig. 10), the further analysis is restricted to a normally incident acoustic wave. Figure 15 shows the sound power $\Pi$ radiated by the ETICS wall with DVAs, employing Eq. 6. The sound power radiated by the ETICS wall without DVAs is shown as a reference.

The reduction of the radiated sound power around $320 \mathrm{~Hz}$, i.e., the resonance frequency of the DVA's on a fixed base, is evident. This is a known behaviour of DVA's: at the (fixed-base) resonance frequency of the DVA a vibration reduction of the structure to which the DVA is attached, is obtained. However, this happens at the expense of an increased vibration, and hence radiated sound power, below and above the (fixed-base) DVA resonance frequency. In this case, at $250 \mathrm{~Hz}$ the seismic mass is resonating in-phase with the wall, while at $385 \mathrm{~Hz}$ the seismic mass is resonating out-of-phase with respect to the wall.

Fig. 15 shows the sound power $\Pi$ computed from the velocity field including and excluding the motions of the seismic mass. In the latter case the sound radiation of the seismic mass was not taken into account. In the frequency region around $250 \mathrm{~Hz}$ (plaster and seismic mass vibrating in-phase) the seismic mass contributes to the radiated sound power, while in the frequency region around $385 \mathrm{~Hz}$ (plaster and seismic mass vibrating out-of-phase) the seismic mass reduces the radiated sound power due to constructive interference.

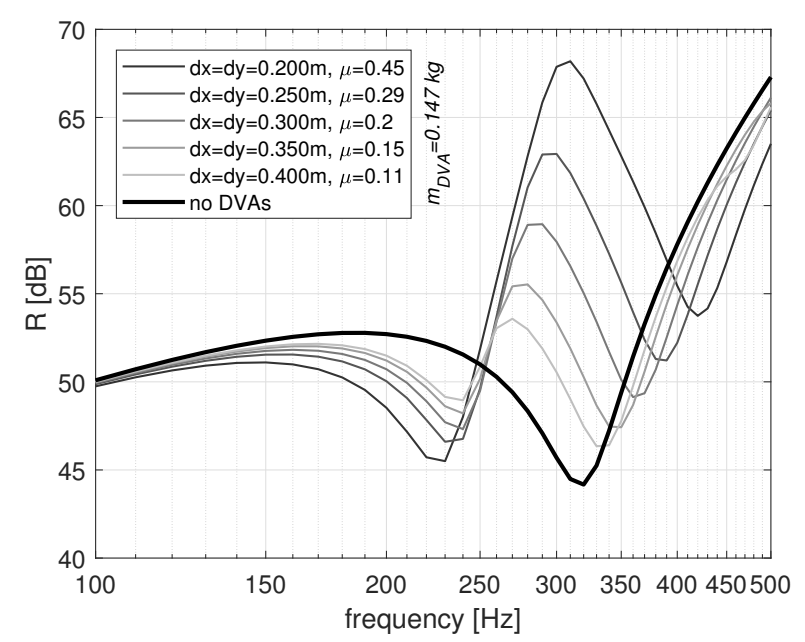

(a)

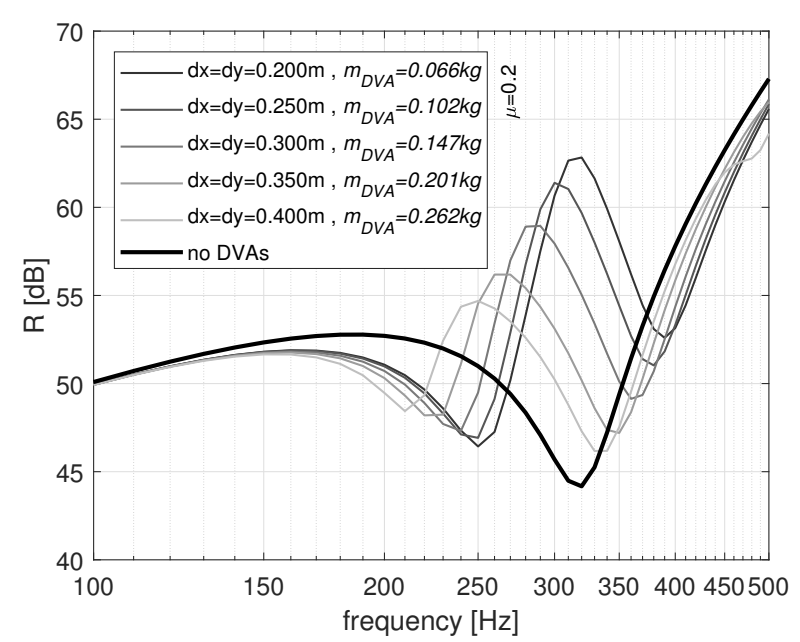

(b)

Figure 16: Numerically predicted sound reduction index $R$ for normally incident $(\beta=0 \mathrm{rad})$ airborne sound, for a DVA resonance frequency $f_{D V A}=320 \mathrm{~Hz}$, and for varying DVA inter-spacing. (a) Fixed seismic mass $m=0.146 \mathrm{~kg}$. (b) Fixed mass ratio $\mu_{s c}=0.45 \%, \mu_{m l}$ $=17 \%$, corresponding to a modal mass ratio $\mu=20 \%$.

Figure 16 a depicts the numerically predicted sound reduction index $R$ for normally indicent airborne sound for different inter-distances and a fixed $m_{D V A}$ and varying the inter-distances $\left\{d_{x}, d_{y}\right\}$. The smaller the inter-distances $\left\{d_{x}, d_{y}\right\}$, the higher the sound reduction index $R$ around $320 \mathrm{~Hz}$, as a result of the larger modal mass ratio $\mu$. In Fig. $16 \mathrm{~b}$ the inter-distance is varied while keeping the modal mass-ratio $\mu\left(\approx \mu_{m l}\right)$ constant. From this figure it can be concluded that the inter-distances $\left\{d_{x}, d_{y}\right\}$ should be chosen wisely. A very small inter-distance leads to good results, but a large number of DVAs is impractical.

The span of control is defined as the spatial region for which a reduction in the vibration levels can be obtained. 
For too large inter-distances the performance of the DVAs diminishes, as a consequence of the limited span of control of the DVAs (see also discussion on Fig. 13 earlier in this section).

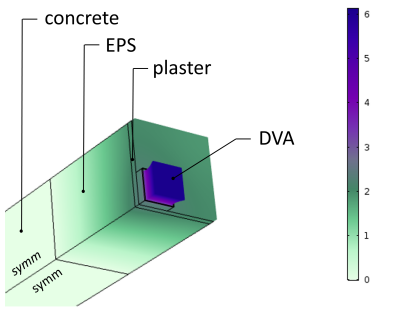

(a) $d_{x}=d_{y}=0.2 \mathrm{~m}$ $m_{D V A}=0.0655 \mathrm{~kg}, f_{1}=254.2+$ $18.7 \mathrm{i} \mathrm{Hz}(\eta=7.4 \%)$.

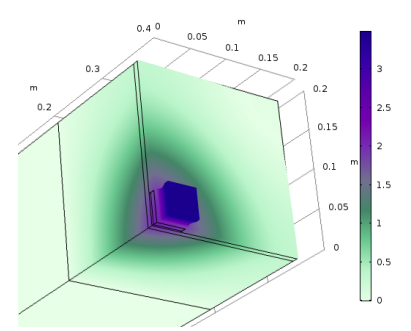

(c) $d_{x}=d_{y}=0.4 \mathrm{~m}$ $m_{D V A}=0.262 \mathrm{~kg}, f_{1}=216.0+$ $14.3 \mathrm{i} \mathrm{Hz}(\eta=6.6 \%)$

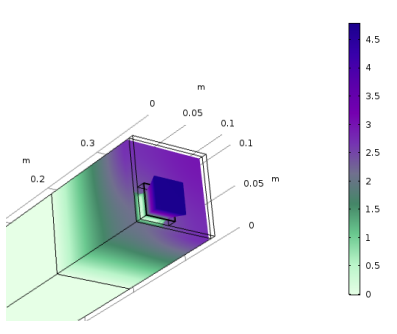

(b) $\quad d_{x}=d_{y}=0.2 \mathrm{~m}$, $m_{D V A}=0.0655 \mathrm{~kg}, f_{2}=387.8+$ $28.3 \mathrm{i} \mathrm{Hz}(\eta=7.3 \%)$.

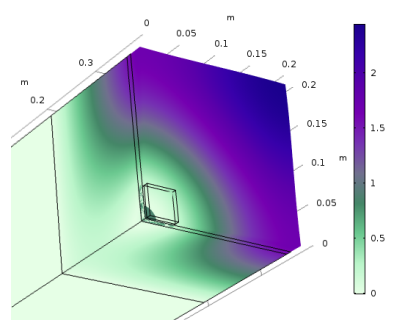

(d) $d_{x}=d_{y}=0.4 \mathrm{~m}$, $m_{D V A}=0.262 \mathrm{~kg}, f_{2}=334.6+$ $23.7 \mathrm{i} \mathrm{Hz}(\eta=7.1 \%)$
Figure 17: Numerically predicted eigenmodes (deformed shapes) of ETICS wall with DVAs, Bloch-Floquet boundary conditions corresponding to $\beta=0$ rad (yielding $z$-direction motions only), $f_{D V A}=320 \mathrm{~Hz}$. In all cases $\mu_{s c}=0.45 \%, \mu_{m l}=17 \%$, corresponding to a modal mass ratio $\mu=20 \%$. Color scale indicates the vibration amplitude. Only one quarter of the model shown.

The effect of the limited span of control is illustrated in more detail in Fig. 17, which shows the two coupled eigenmodes of the ETICS wall with DVA for a small and a large inter-spacing $\left\{d_{x}, d_{y}\right\}$, keeping the modal mass ratio $\mu$ constant (as in Fig 16b). The span of control is limited due to the finite (low) stiffness of the $7 \mathrm{~mm}$ layer of reinforced adhesive mortar. For the inter-distance $d_{x}=d_{y}=0.2 \mathrm{~m}$, the first two eigenfrequencies occur at $254 \mathrm{~Hz}$ and $388 \mathrm{~Hz}$, corresponding to the two dips in the sound reduction index $R$ shown in Fig. 16b for this case. For the inter-distance $d x=d y=0.4 \mathrm{~m}$, the first two eigenfrequencies occur at significantly lower frequencies; $216 \mathrm{~Hz}$ and $335 \mathrm{~Hz}$. Also at these eigenfrequencies a dip in the sound reduction index $R$ can be observed. The reason why the eigenfrequencies shift down in frequency when increasing the inter-spacing $\left\{d_{x}, d_{y}\right\}$ is that the the stiffness of the layer of reinforced adhesive mortar is decreasing due to its local deformation as illustrated in Fig. 17c and 17d. These effects are related to the reduction of the span of control of the DVAs with increasing inter-spacing causing a shift of the frequency with maximum impact on the sound reduction index $R$ towards lower frequencies. Using a too large inter-spacing also reduces the performance of the DVAs, as results from the deformation pattern shown in Fig. 17c,17d.

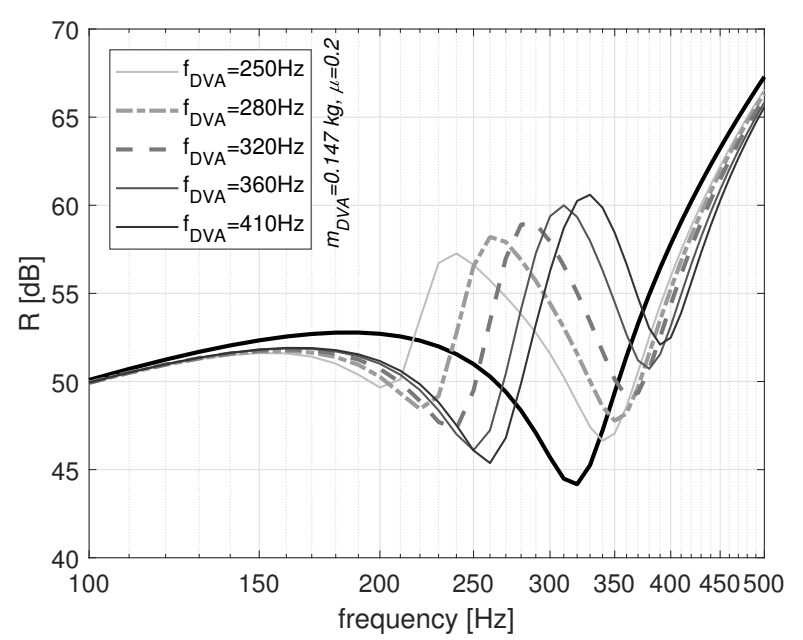

Figure 18: Numerically predicted sound reduction index $R$ for normally incident $(\beta=0 \mathrm{rad})$ airborne sound, a fixed seismic mass $m_{D V A}=0.146 \mathrm{~kg}, d_{x}=d_{y}=0.3 \mathrm{~m}$, modal mass ratio $\mu=20 \%$, and varying resonance frequency $f_{D V A}$

In the analysis discussed above, the DVAs were tuned at $f_{D V A}=320 \mathrm{~Hz}$, equal to the ETICS mass-spring-mass resonance $f_{0}$. As mentioned above, according to Ormondroyd and Den Hartog [11] the resonance frequency of the DVA should be tuned slightly below the natural frequency of the host structure $f_{0}: f_{D V A}=f_{0} /(1+\mu)$. This implies that the optimal tuning frequency equals $320 /(1+0.2)$ $=270 \mathrm{~Hz}$. In order to study the impact of the resonance frequency of the DVA on the noise reduction index $R$, an additional analysis was performed, as discussed below.

Considering the results shown in Fig. 18 a tuning of the DVA to $320 \mathrm{~Hz}$ might be seen as an optimum tuning frequency, as this tuning gives the optimum increase of $R$ at the frequency where the ETICS system has a minimum. However, in the original optimisation used by Omondroyd and Den Hartog, the criterion to choose the optimal value for $f_{D V A}$ was to tune the DVA in such a way that the magnitude of the displacement response at the two dips in $R$ that are caused by the two resonances of the coupled DVAwall system are equally low [35]. This occurs at a tuning frequency between $f_{D V A}=280 \mathrm{~Hz}$ (dash-dotted cure) and $320 \mathrm{~Hz}$ (dashed curve). However, the limited span of control of the DVAs also causes a shift of the frequency with maximum impact on the sound reduction index $R$ towards lower frequencies, as explained in the previous paragraph.

It is also true that for practical reasons, the interdistances $\left\{d_{x}, d_{y}\right\}$ are preferred to be as large as possible in order to reduce the number of DVAs required. In addition, the negative effects of the DVAs dips in $R$ caused by the two resonances of the coupled system should not be too deep, whilst at the ETICS resonance frequency the sound reduction index $R$ should preferably be as high as possible. Taking into account all these considerations, the interdistance $\left\{d_{x}, d_{y}\right\}=\{0.31,0.28\} \mathrm{m}$ is considered the maxi- 
mum distance and a seismic mass $m_{D V A}=0.146 \mathrm{~kg}$ (corresponding to a modal mass-ratio $\mu=20 \%, \mu_{s c}=0.45 \%, \mu_{m l}$ $=17 \%)$ is considered the minimum weight of the seismic mass that would allow for reasonable effects of the DVAs. According to the classical tuning laws the optimal damping reads $\eta_{D V A}=\sqrt{3 \mu /\left(4(1+\mu)^{3}\right)}$, which gives $\eta_{D V A} \approx 0.3$.

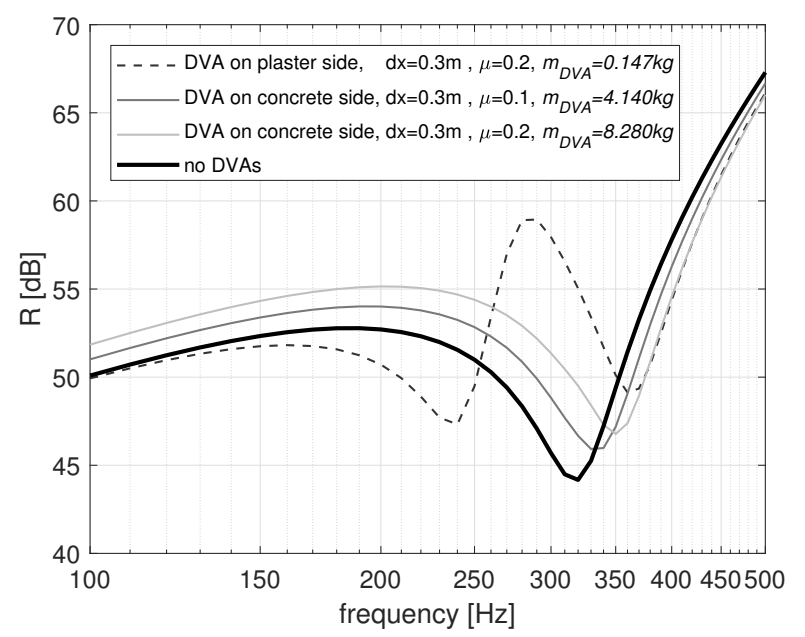

Figure 19: Numerically predicted sound reduction index $R$ for normal incident $(\beta=0 \mathrm{rad})$ plane wave acoustic excitation. Solid curves: DVAs mounted at silicate cement wall side. Dashed curve: DVAs mounted at plaster side.

Needless to say that the DVAs should be mounted on the plaster side, and not on the base wall of the massive ETICS wall. For the sake of illustration, the numerical simulations were repeated for a case in which the DVAs are mounted at the silicate cement wall side. Fig. 19 shows that the seismic mass of the DVAs need to be much higher when mounted on the silicate cement wall side. Actually, the mass of each single DVA needs to be $8.28 \mathrm{~kg}$ for $\left\{d_{x}, d_{y}\right\}=0.3 \mathrm{~m}, \mu=0.2$. Since the sound reduction index below the coincidence region is strongly influenced by the mass per unit area of the wall, the added mass also gives a slightly higher sound reduction index $R$ at lower frequencies $\left(20 \cdot \log _{10}(1.2) \approx 1.6 \mathrm{~dB}\right)$.

\section{Tuning and manufacturing of the DVAs}

As the measurements presented in Section 2 showed that the ETICS-dip occurs in the frequency range 250 - 400 $\mathrm{Hz}$, DVAs were designed and constructed to compensate as much as possible for the reduced noise reduction in$\operatorname{dex} R$ in this frequency range. In the previous section minimum requirements for the DVAs were derived on theoretical grounds. However, in real life, the realisation of a DVA based on commercial items is bounded by practical constraints like the available types of materials for the spring, and the available dimensions (and thus weights) of available seismic masses.

During the experimental campaign two DVA-types were tested. Both types of DVA consisted of an elastic material

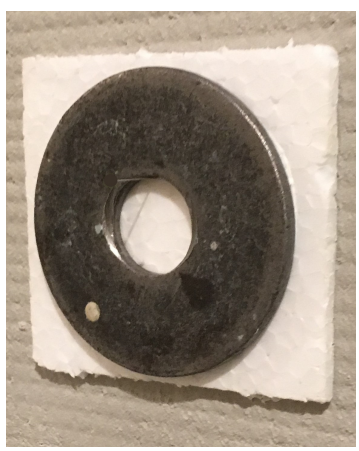

(a) EPS based DVA Type 1

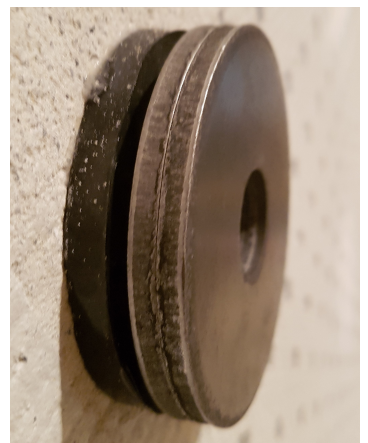

(b) Sorbothane based DVA Type $2 \mathrm{a}$

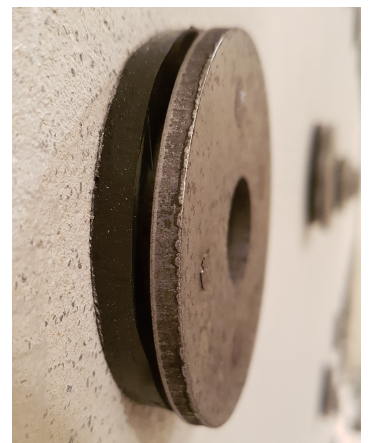

(c) Sorbothane based DVA Type $2 \mathrm{~b}$
Figure 20: DVA Types 1, 2a and 2b.

and a seismic mass. The elastic materials used were respectively Expanded Polystyrene (EPS) and Sorbothane (from Sorbothane, Inc., Kent, Ohio, USA). The seismic mass consisted in an assembly of so-called "Fender" washers. Details of the EPS based DVA, denoted as DVA Type 1, are listed in Table 2. The Sorbothane-based DVA was tested in two configurations, denoted by DVA Type $2 \mathrm{a}$ and type $2 \mathrm{~b}$. These differed in the weight of the seismic mass (See Table 2 for details). A picture of the different types of DVA is shown in Figure 20.

DVA Type 1 contained only 1 Fender washer as seismic mass. DVA Type 2a and type $2 \mathrm{~b}$ contained a seismic mass that was composed of a number of Fender washers. The interface washer (i.e the one in contact with the Sorbothane material) was the same for both the type 2 DVAs, having an inner diameter of $20 \mathrm{~mm}$ and an outer diameter of $54 \mathrm{~mm}$. In addition to the interface washer, Type $2 \mathrm{a}$ had two Fender washers with dimension $80 \mathrm{~mm}$ outer, $22 \mathrm{~mm}$ inner diameter, $5 \mathrm{~mm}$ thickness. Figure 21a shows a number of assemblies of this type. Type $2 \mathrm{~b}$ contained only one additional Fender washer of the same type. The washers were glued together by means of Loctite 415 . Sorbothane material is quite sticky and self-adhesive, which allowed the washers to be mounted to the disk without using glue.

\subsection{Modal analysis results}

Modal analysis tests were performed to determine the fundamental resonance frequencies and the loss factors of 


\begin{tabular}{|l|l|l|l|}
\hline & Type 1 & Type 2a & Type 2b \\
\hline Elastic material & EPS & Sorbothane 30 DURO Black \\
thickness & $5 \mathrm{~mm}$ & $6.35 \mathrm{~mm}(0.25 ")$ \\
diameter & & $76.2 \mathrm{~mm}\left(3^{\prime \prime}\right)$ \\
\hline Seismic mass & 1 washer & $1+2=3$ washers & $1+1=2$ washers \\
total weight & $146 \mathrm{~g}$ & $410 \mathrm{~g}$ & $230 \mathrm{~g}$ \\
interface washer & none & $\varnothing d_{\text {in }}=20 \mathrm{~mm}, \varnothing d_{\text {out }}=54 \mathrm{~mm}$ \\
\hline
\end{tabular}

Table 2: Details of DVA Type 1, type 2a and type $2 \mathrm{~b}$

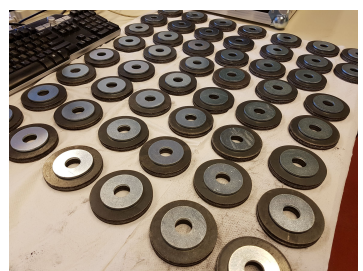

(a) Assembled seismic masses for Sorbothane based DVAs Type 2a. The interface washers on top.

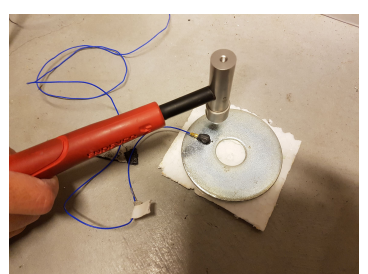

(b) Modal analysis testing of EPS based DVA on a rigid foundation.
Figure 21: Assembled seismic masses and modal analysis testing.

the different types of DVAs. The spring-element of the DVA was rigidly mounted on a heavy, thick floor (Fig. 21b). The measured transfer functions, from hammer force to acceleration, are shown in Fig. 22. The loss factors $\eta$ of the fundamental resonance frequencies of the two DVA types were determined using the half power bandwidth approach [36].

The resulting eigenfrequencies and loss factors are summarized in Table 3. It turns out that Sorbothane based DVAs are much more damped than the EPS based ones. The high damping of Sorbothane was the main reason to select this material as elastic layer of Type 2 DVAs. Note that the squared ratio of the eigenfrequencies of DVA Type $2 \mathrm{a}$ and Type $2 \mathrm{~b}\left((426 \mathrm{~Hz} / 323 \mathrm{~Hz})^{2}=1.74\right)$ corresponds well with the ratio of the seismic masses of Type $2 \mathrm{a}$ and Type $2 \mathrm{~b}(0.410 \mathrm{~kg} / 0.230 \mathrm{~kg}=1.78)$, thus confirming, to some extent, the linearity of the Sorbothane spring.

As concluded in theoretical Section 3.2, the maximum allowable inter-distance equals $\left\{d_{x}, d_{y}\right\}=\{0.31,0.28\} \mathrm{m}$, and the minimum weight of the seismic mass equals $m_{D V A}=0.146 \mathrm{~kg}$ (corresponding to a modal mass-ratio $\left.\mu=20 \%, \mu_{s c}=0.45 \%, \mu_{m l}=17 \%\right)$. The optimal DVA

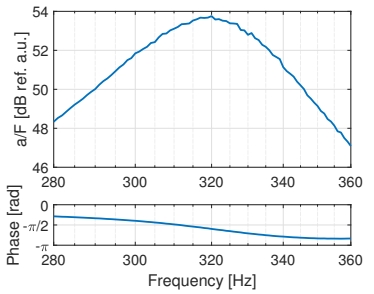

(a) EPS based DVA Type 1

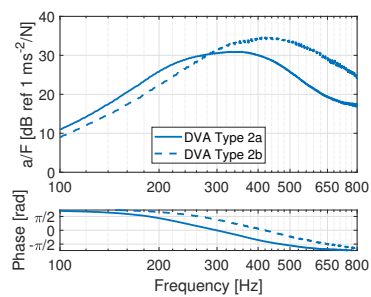

(b) Sorbothane based DVA Type $2 \mathrm{a}$ and Type $2 \mathrm{~b}$
Figure 22: Modal analysis transfer functions, DVA on rigid foundation

\begin{tabular}{|l|l|l|}
\hline DVA Type & Eigenfrequency $[\mathrm{Hz}]$ & Loss factor $\eta[-]$ \\
\hline 1 & 319 & 0.157 \\
\hline $2 \mathrm{a}$ & 323 & 0.73 \\
\hline $2 \mathrm{~b}$ & 426 & 0.61 \\
\hline
\end{tabular}

Table 3: Eigenfrequencies and loss factors of DVA Type 1, 2a and $2 \mathrm{~b}$.

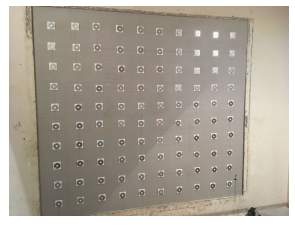

(a) 100 DVAs

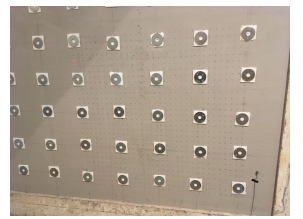

(b) 100 DVAs, detailed view $L D V$ scan area
Figure 23: EPS based DVAs Type 1 mounted on the wall

natural frequency is about $f_{D V A}=320 \mathrm{~Hz}$, and the optimal loss factor of the DVA equals $\eta_{D V A}=0.3$. To this respect, Type 1 DVA satisfies the natural resonance frequency very well. However, its damping is too low (see Table 3), as compared to the optimum predicted by the classical tuning law. DVA Type 2, on the other hand, has a bit too high amount of damping. Its natural resonance frequency is fair for Type $2 \mathrm{a}$ and a bit too high for Type $2 \mathrm{~b}$. The seismic mass of Type 1 DVA (146 gram, see Table 2) is equal to the minimum weight from a theoretical point of view. The seismic mass of Type 2b DVA (230 gram) is a bit too high, and the seismic mass of DVA Type 2a (410 gram) is much higher than the minimum weight given in Section 3.2. It should be noted that a higher seismic mass gives, according to the predictions, a bigger impact on the sound reduction index $R$.

The DVAs were mounted on the plaster side of the ETICS wall as suggested in the previous section. The inter-spacing of the DVAs was gradually varied, starting from a course grid up to a fine grid, allowing to investigate the effect of the inter-spacing from an experimental point of view. The DVAs were mounted along a regular grid, as shown in Fig. 23 and Fig. 24. An interspacing $\left\{d_{x}, d_{y}\right\}$ between the DVAs of $64 \mathrm{~cm}, 64 \mathrm{~cm}$ interlaced (resulting in an effective interspacing of $d_{x}=d_{y}=0.64 \mathrm{~m} / \sqrt{2}=0.45 \mathrm{~m}$ ) and $32 \mathrm{~cm}$ was used for the $5 \times 5=25$ DVAs, $10 \times 10$ interlaced $=50$ DVAs and $10 \times 10=100$ DVAs configuration, respectively. The configurations tested are summarized in Table 4.

\section{Measurement results on ETICS wall with DVAs}

Measurements on the ETICS wall were conducted to determine the effect of the DVAs in terms of vibrational and acoustic response to airborne sound. The wall was exposed to pink noise in the sending room of a transmission loss facility, as described in Section 2. The vibrational response was measured by means of a scanning laser Doppler 
(a) 25 DVAs

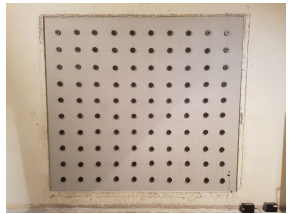

(c) 100 DVAs

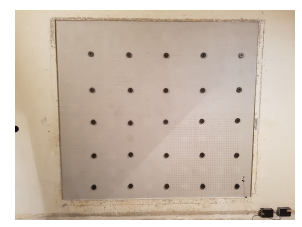

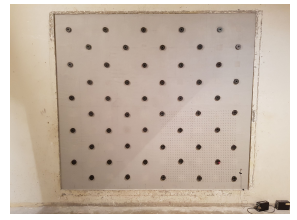

(b) 50 DVAs

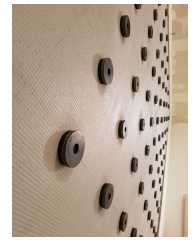

(d) 100 DVAs
Figure 24: Sorbothane based DVAs Type 2 mounted on the wall

\begin{tabular}{|l|l|l|l|l|}
\hline DVA & \# of DVAs & $d_{x}, d_{y}$ & $\begin{array}{l}\mu_{s c} \\
(\text { Eq.12) }\end{array}$ & $\begin{array}{l}\mu_{m l} \\
(\text { Eq.13) }\end{array}$ \\
\hline Type 1 & $25(5 \times 5)$ & $0.64 \mathrm{~m}$ & $0.1 \%$ & $3.5 \%$ \\
$(146 \mathrm{~g})$ & $50(10 \times 10$ interlaced $)$ & $0.45 \mathrm{~m}$ & $0.2 \%$ & $7 \%$ \\
& $100(10 \times 10)$ & $0.32 \mathrm{~m}$ & $0.4 \%$ & $14 \%$ \\
\hline Type 2a & $25(5 \times 5)$ & $0.64 \mathrm{~m}$ & $0.3 \%$ & $10 \%$ \\
$(410 \mathrm{~g})$ & $50(10 \times 10$ interlaced $)$ & $0.45 \mathrm{~m}$ & $0.5 \%$ & $20 \%$ \\
& $100(10 \times 10)$ & $0.32 \mathrm{~m}$ & $1 \%$ & $39 \%$ \\
\hline Type 2b & $100(10 \times 10)$ & $0.32 \mathrm{~m}$ & $0.6 \%$ & $22 \%$ \\
$(230 \mathrm{~g})$ & & & & \\
\hline
\end{tabular}

Table 4: DVA configurations tested.

measurement system (discussed in Section 5.1). The noise reduction index $R$ was measured according to ISO 10140-2 (discussed in Section 5.2).

\subsection{Laser Doppler vibrometry results}

The response of the ETICS wall was measured at the receiving side of the structure by means of an in-house developed scanning laser Doppler vibrometer system comprising a single head laser Doppler vibrometer, Polytec OFV-505, a Polytec controller OFV-5000, and a dual-axis scanning mirror system from Thorlabs. The vibrational response was measured along an area of $1.32 \mathrm{~m} \times 1.20 \mathrm{~m}$, in the bottom right corner of the $3.30 \mathrm{~m} \times 3.00 \mathrm{~m}$ wall, as shown in Fig. 25. A limited portion of the ETICS wall was measured to reduce the measurement time. The vibrational response was measured every $4 \mathrm{~cm}$ along a $2 \mathrm{D}$ grid of 33 (horizontal) $\times 30$ (vertical) points, $n=990$ points in total. The response of each DVA in the measurement area was measured as well using 4 points per seismic mass. Signals were recorded at a sampling rate of $44.1 \mathrm{kHz}$. As the highest frequency of interest of the LDV measurements is especially around the eigenfrequency of the DVAs, the data could be downsampled to $4.41 \mathrm{kHz}$ before processing in order to reduce the amount of storage required. An accelerometer at a fixed position on the wall (plaster side), in the bottom right, about $15 \mathrm{~cm}$ from the edges, was used as a reference signal $r$ to reconstruct the phase of the response. The frequency response function $H_{r i}$ between the response of the structure at point $i, i=1 \ldots n$, as measured by the LDV, relative to the reference signal $r$, can be estimated using the optimal estimate [37]:

$$
H_{i r}(\omega)=\frac{S_{i r}(\omega)}{S_{r r}(\omega)}
$$

where the cross-spectra between the response of the structure at $n$ positions and the reference signal are denoted by $S_{i r}(\omega)$, and the autospectrum of the reference signal by $S_{r r}(\omega)$. The estimates for $S_{i r}(\omega)$ contain the phase information between the responses at points $i$, with $i=1 \ldots n$, and the reference response at point $r$, from which the mutual phase differences between the responses at pairs of points $i$ and $j$, with $i, j=1 \ldots n$, can be determined as

$$
H_{i j}(\omega)=\frac{S_{i r}(\omega)}{S_{j r}(\omega)}
$$

The autospectrum of the reference signal can be estimated by taking the average from the $n$ measurement passes [38],

$$
\left\langle S_{r r}\right\rangle_{n}=\frac{1}{n} \sum_{i=1}^{n} S_{r r(i)},
$$

yielding an improved signal to noise ratio as compared to each individual autospectrum $S_{r r(i)}$ (theoretically a factor $\sqrt{n})[39,38]$. In combination with the frequency response functions $H_{r i}$ for different locations $i$, the averaged velocity response function $v_{i}$ (units: $\left[\mathrm{ms}^{-1}\right]$ ) at each individual point $i$, can be estimated as [38]:

$$
v_{i}=H_{r i} \sqrt{\left\langle S_{r r}\right\rangle_{n}}
$$

Each transfer function $H_{r i}$ in Eq. 15 was computed using a record length of $2.6 \mathrm{~s}$ (yielding a frequency resolution $\mathrm{df}=0.385 \mathrm{~Hz}$ ), 16 averages with $75 \%$ overlap (total measurement time $13 \mathrm{~s}$ ) and using a Hanning window. The total measurement time for the set of $n=990$ points, adopted for the measurements, was somewhat less than 6 hours (including the transfer time of the measurement data and the waiting time to stabilize the mirrors). The computed velocity response $v_{i}$ at each point on the surface of the wall (Eq. 17), including its phase information, forms the basis of the following analysis, in which emphasis is put on the results obtained for DVA Type 2. In Section 5.2 the ISO 10140-2 results are presented for both DVA types 1 and 2 .

The operational deflection shapes of three configurations tested (ETICS wall without DVAs, ETICS wall with 50 DVAs Type 2a and ETICS wall with 100 DVAs Type 2a) are shown in Fig. 26 for a single narrow band frequency close to the ETICS mass-spring-mass resonance frequency. The DVAs clearly reduce the vibration levels.

Figure 27 presents the spatially averaged vibration level for the ETICS wall and for the ETICS wall with Type 2 DVAs as measured by the LDV. The $1 / 12^{\text {th }}$ octave band spectra show that, for Type 2a DVA, the reduction in the 


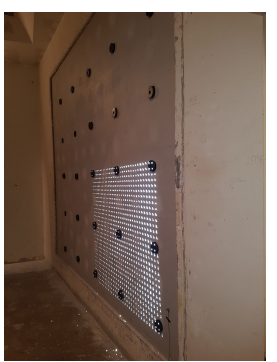

(a) 25 DVAs (b) 100 DVAs

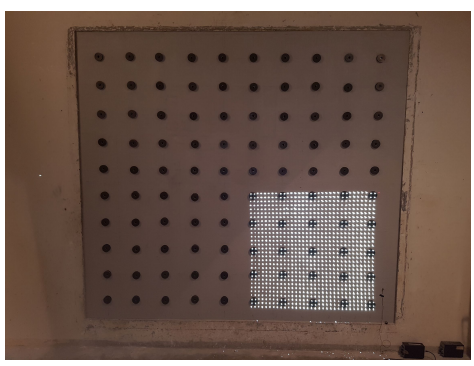

Figure 25: LDV scan area. Retro-reflecting pieces of tape used to increase the optical reflection are clearly visible due to camera flash.

vibration level is roughly occurring in the frequency range from $250 \mathrm{~Hz}$ up to $320 \mathrm{~Hz}$. This frequency range is below the damped resonance frequency of Type $2 \mathrm{a}$ DVA, which is about $323 \mathrm{~Hz}$. This effect can be a consequence of the very high loss factor $\eta=0.73$ of this configuration (see Table 3 ) It can also be caused by the limited span of control which causes the frequency with maximum impact on the sound reduction index $R$ to be shifted towards lower frequencies, as observed in the numerical simulations (Section 3.2).

Since Type 2a DVA acts at lower frequencies than anticipated, it was decided to test another configuration, Type 2b DVA, having a higher eigenfrequency. The latter was realised by reducing the seismic mass of the DVA (see Table 2 for details). As expected, in Fig. 27 (dashed curve), the maximum response occurs at a higher frequency, which is slightly better tuned to the ETICS resonance dip, yielding a reduction of the spatially averaged vibration levels $\Delta L_{V}$ of $10 \mathrm{~dB}$ at $300 \mathrm{~Hz}$.

The ratio of the vibration levels of the DVA seismic mass to the vibrations of the wall just around a DVA was computed by taking the average (complex) value of the transfer functions at 4 positions distributed on the seismic mass of one DVA and considering the spatial average of 20 measurement points placed around the DVA. The resulting transfer functions are shown in Fig. 28. At very low $(<100 \mathrm{~Hz})$ and very high $(>500 \mathrm{~Hz})$ frequencies, the vibration levels of the DVA seismic mass are the same as the vibrations levels of the plaster wall $\left(\Delta \mathrm{L}_{V} \approx 0\right)$. Figure $28 \mathrm{~b}$ and $28 \mathrm{c}$ show that below approximately $280 \mathrm{~Hz}$ the movements of the DVA seismic mass and the wall are in phase and $\Delta \mathrm{L}_{V}$ is positive, meaning that the DVA seismic masses are vibrating with a higher amplitude than the wall. This experimental observation is in full agreement with the numerical simulation results shown in Fig. 12 (Section 3.2). Above approximately $280 \mathrm{~Hz}, \Delta \mathrm{L}_{V}$ is negative, meaning that the DVA seismic mass is vibrating at a lower level as compared to the wall, which is also in agreement with the results shown in Fig. 13.

As the DVAs can have vibration levels that are higher than the ETICS wall at specific frequencies, in the computation of the spatially averaged vibration levels of the plaster from the LDV data, the vibrations of the DVA seismic masses were discarded. The results (Fig. 29) show a

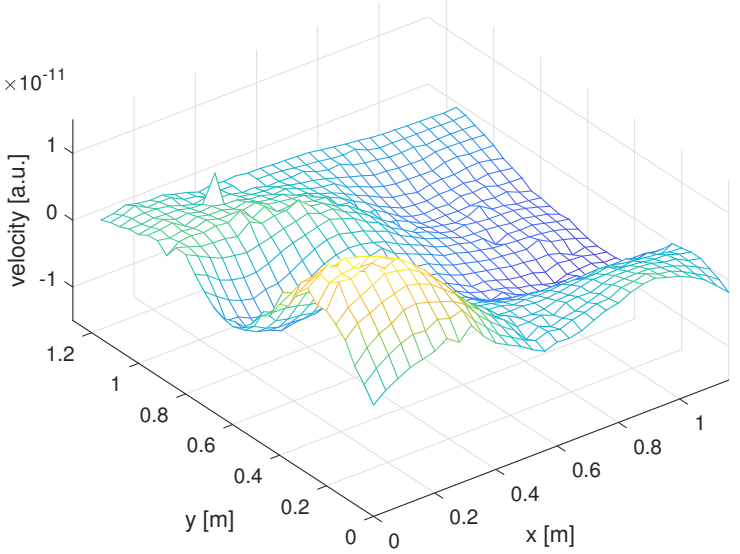

(a) wall+ETICS

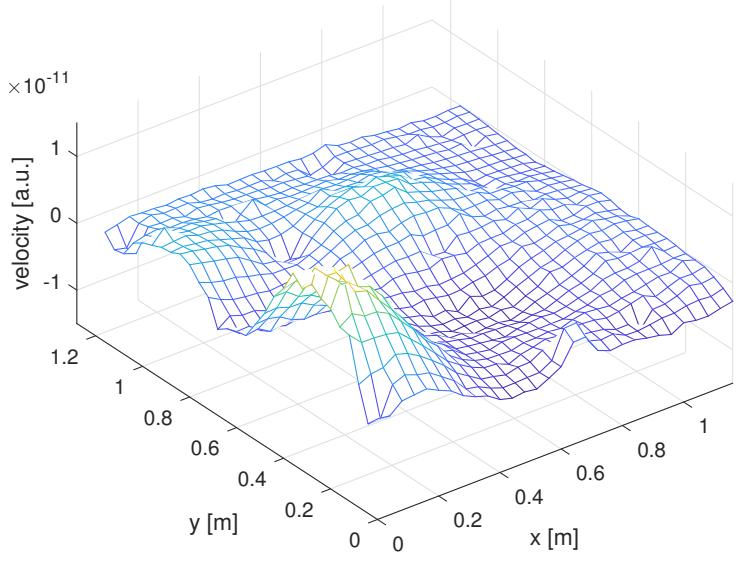

(b) wall+ETICS+50 DVAs Type $2 \mathrm{a}$

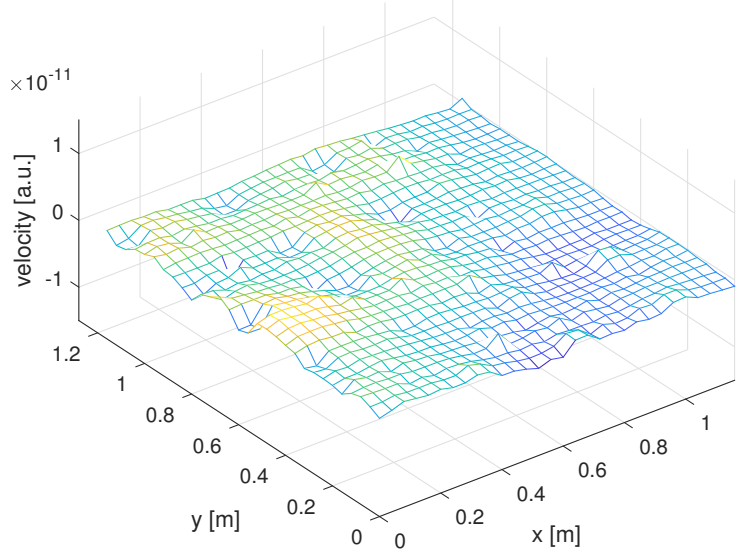

(c) wall+ETICS+100 DVAs Type 2a

Figure 26: Operational deflection shapes at $297 \mathrm{~Hz}$ (narrowband FFT result), for different wall configurations. 


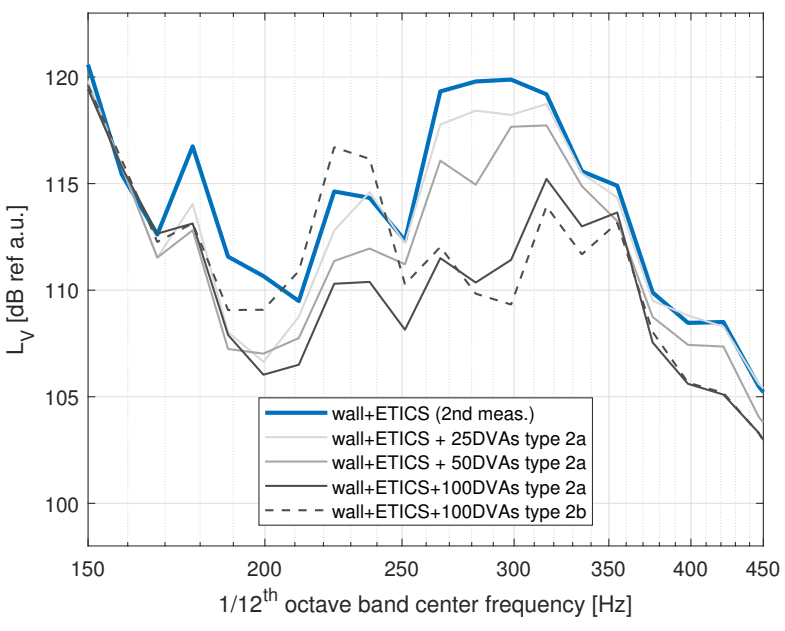

(a)

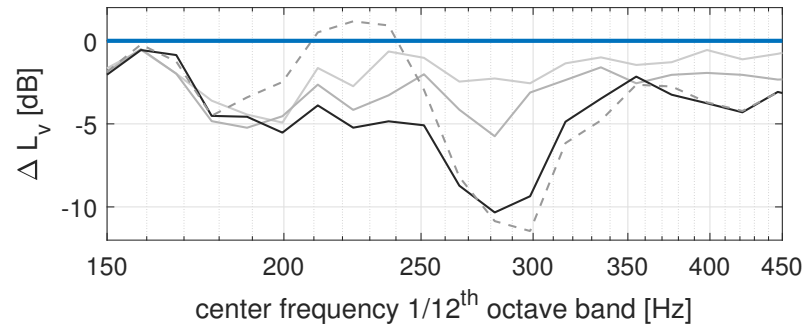

(b)

Figure 27: Spatially averaged vibration levels $\mathrm{L}_{V}$ and vibration level differences $\Delta \mathrm{L}_{V}$ of the plaster wall, excluding the DVA's. a) $\mathrm{L}_{V}$ as measured per case. b) Level difference $\Delta \mathrm{L}_{V}$ relative to the case (wall+ETICS).

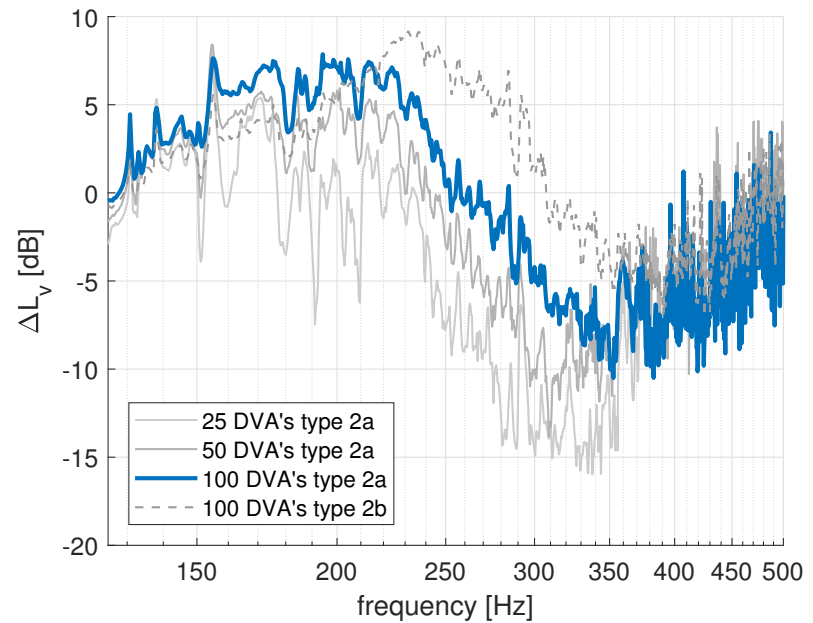

(a) Magnitude

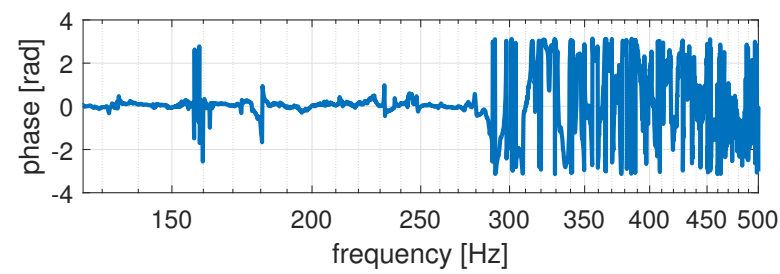

(b) Phase

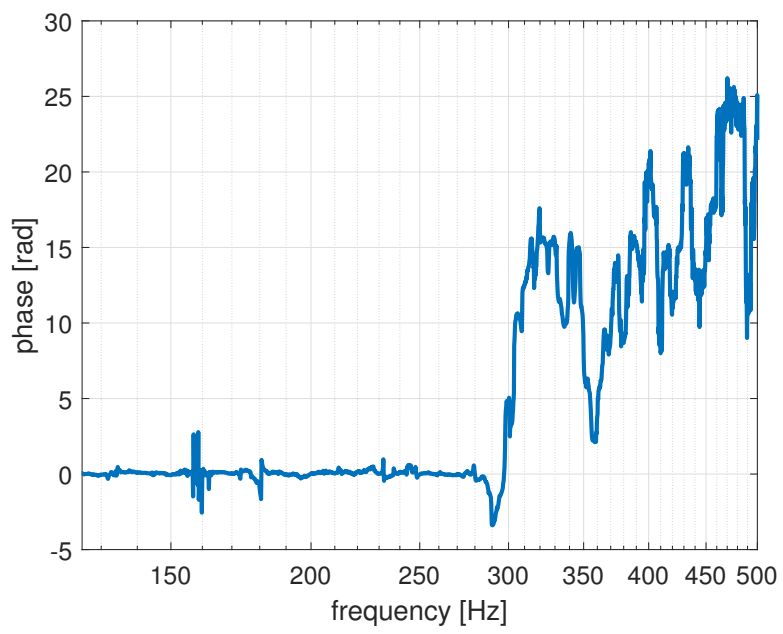

(c) Unwrapped phase

Figure 28: Vibration of the DVA seismic mass relative to the vibrations of the wall, narrowband FFT spectra. Solid thick curves: wall with 100 DVAs Type 2. a): Level difference. b) Phase difference. c) Unwrapped phase difference. 


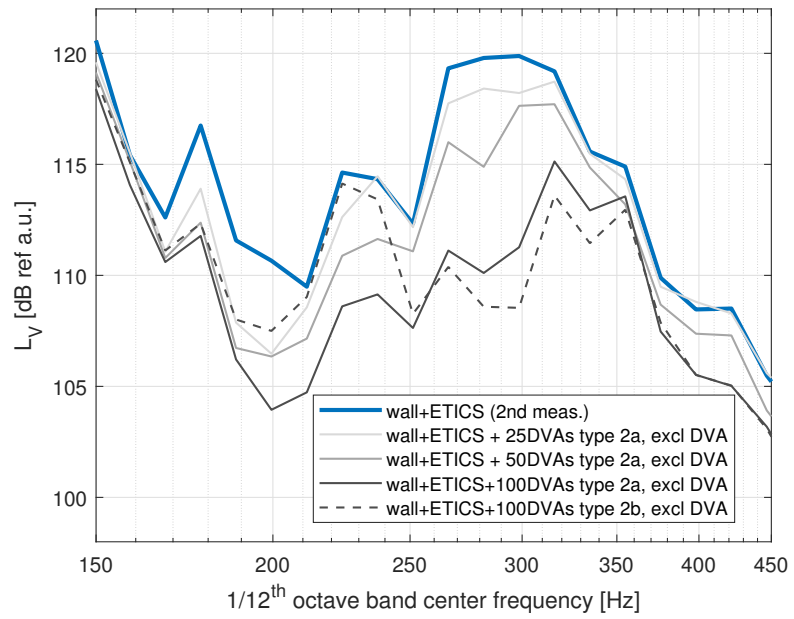

(a)

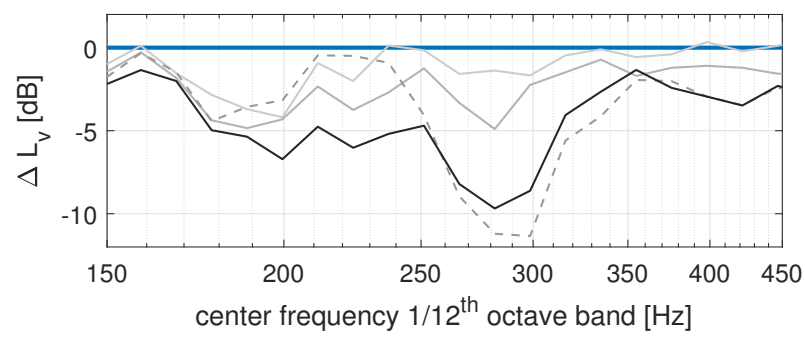

(b)

Figure 29: Spatially averaged vibration levels $\mathrm{L}_{V}$ and vibration level differences $\Delta \mathrm{L}_{V}$, computed from LDV data, excluding the DVAs. a) $\mathrm{L}_{V}$ as measured per case. b) Level difference $\Delta \mathrm{L}_{V}$ relative to the case (wall+ETICS). lower vibration level than the one given in Fig. 27, especially in the frequency range from $200-280 \mathrm{~Hz}$. In effect, omitting the DVA seismic masses and considering the vibrations of the plaster layer only, the configuration of 100 DVAs Type $2 \mathrm{~b}$ gives a reduction of the spatially averaged vibration levels $\Delta L_{V}$ of more than $10 \mathrm{~dB}$ in the 280 and $300 \mathrm{~Hz} 1 / 12^{t h}$ octave bands (see dashed curve in Fig. 29).

The sound power radiated from the area that was measured by the LDV scanning system can be calculated by evaluating the Rayleigh integral (in spatial domain, not in wavenumber domain as was done in Section 3.1.2) over this area [27]. In this approach the receiving room was assumed to be a semi-infinite acoustic domain and the test wall was baffled. The acoustic pressure $p(\mathbf{r}, \omega)$ at position $\mathbf{r}$ in the acoustic domain, caused by the vibrating surface, can be calculated as

$$
p(\mathbf{r})=\frac{\mathrm{i} \omega \rho}{2 \pi} \iint_{S} v_{\perp}\left(\mathbf{r}_{\mathbf{S}}\right) \frac{e^{-\mathrm{i} k \mathcal{R}}}{\mathcal{R}} d S
$$

where $v_{\perp}\left(\mathbf{r}_{\mathbf{S}}, \omega\right)$ denotes the velocity in the normal direction at position $\mathbf{r}_{\mathbf{S}}$ of the vibrating surface (e.g. $v_{i}$ in Eq. 17), $S$ denotes the area of the vibrating structure, $\mathcal{R}$ is the distance between $\mathbf{r}$ and $\mathbf{r}_{\mathbf{S}}$. Since the normal component of the active acoustic intensity $I$ along the surface $S$ of a vibrating test wall is given by

$$
I\left(\mathbf{r}_{\mathbf{S}}\right)=\frac{1}{2} \mathbb{R e}\left[p\left(\mathbf{r}_{\mathbf{S}}\right) v_{n}^{*}\left(\mathbf{r}_{\mathbf{S}}\right)\right]
$$

where $\mathbb{R e}$ denotes the real part of a complex quantity and the asterisk denotes the complex conjugate, the total radiated active sound power $P$ can be obtained by

$$
\Pi=\iint_{S} I\left(\mathbf{r}_{\mathbf{S}}\right) d S
$$

The computation of $p\left(\mathbf{r}_{\mathbf{S}}, \omega\right)$ involves a proper handling of the singularity in Eq. 18 when $\mathcal{R}=0$, and has been described in many text books (e.g. [40], see also paper [27]). Again, for the sake of brevity, in Eqs. 18,19 and 20 the dependence on the angular frequency $\omega$ was omitted.

The calculated results in Fig. 30c show that the DVAs increase the sound radiation up to $3 \mathrm{~dB}$ in the frequency range from 180 up to $250 \mathrm{~Hz}$ for DVA Type 2a and up to $2.5 \mathrm{~dB}$ in the frequency range from 200 up to $300 \mathrm{~Hz}$ for DVA Type 2b. Because of this contribution to the sound radiation at specific frequencies, it is advisable to incorporate the DVA in the EPS-layer. This concept is recommended to be implemented for further research, so that the sound reduction index will not be adversely affected by the sound radiated by the DVAs at its resonance frequency. This concept was also proposed for the same reason in a theoretical study by Liu et al. [17] for DVAs embedded in sandwich structures. In the determination of the sound reduction index $R$ according to ISO 10140-2 (as discussed in the next section), a correction has been applied to eliminate the sound radiation of the DVAs seismic masses. 


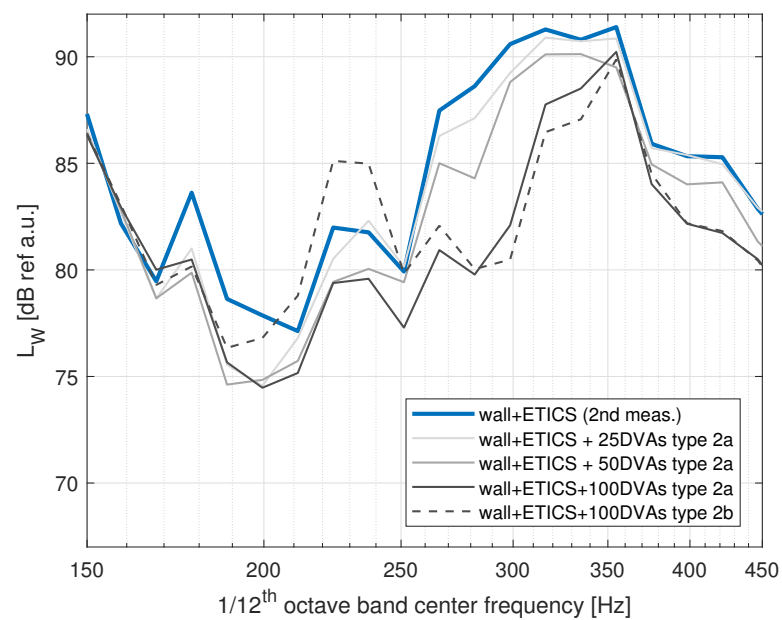

(a)

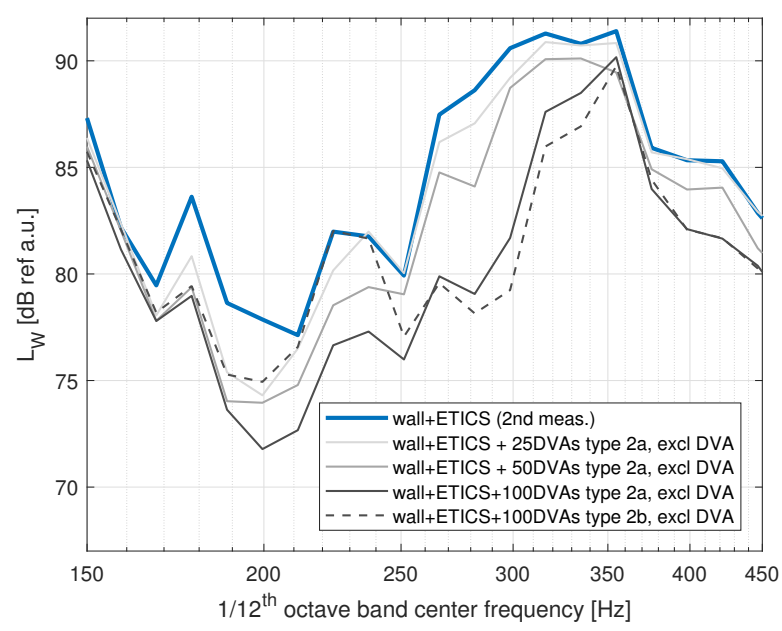

(b)

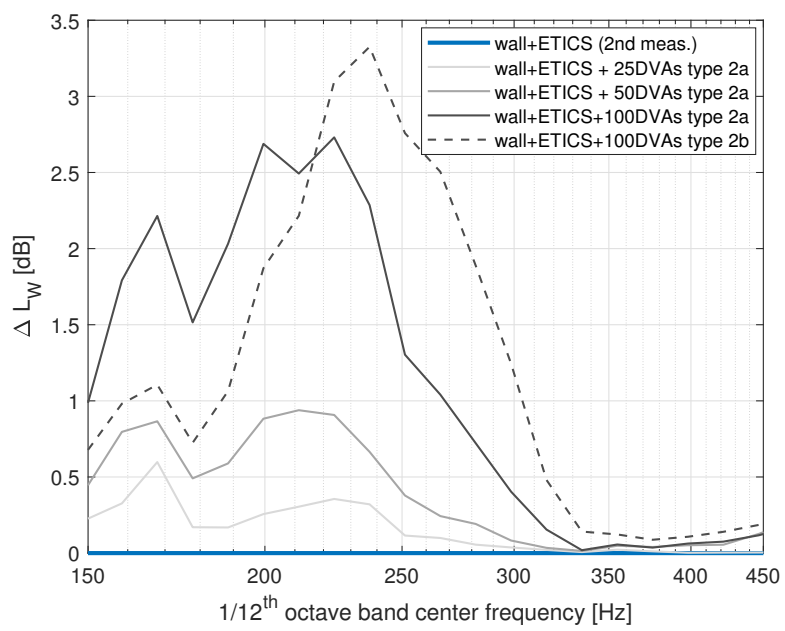

(c)

Figure 30: Active sound power $\mathrm{L}_{W}$, computed from LDV data. (a) including the sound radiation of the DVAs; (b) excluding the sound radiation of the DVA; (c) difference of subplots a and b.
In conclusion, the laser Doppler measurement campaign showed that at specific frequencies a reduction of the spatially averaged vibration level of about $\Delta L_{V}=10 \mathrm{~dB}$ or more (Fig. 27 and 29, DVA Type 2b, $\mu_{s c}=0.6 \%$, $\mu_{m l}=22 \%$ ). Using the measured vibration level as a basis for the computation of the radiated sound power, a reduction of the radiated sound power $\Delta L_{W}$ ranging from 2 to $10 \mathrm{~dB}$ was computed when excluding the sound radiation of the DVA seismic masses (Fig. 30). Including the sound radiation of the seismic masses led to a reduction of $\Delta L_{W}$ up to $3 \mathrm{~dB}$.

\subsection{ISO 10140-2 results}

The results of the measurements performed to assess the sound reduction index $R$ following ISO 10140-2 standard are shown in Fig. 31 and 32 for the EPS based Type 1 DVAs, together with the ETICS-wall as a reference. While the effect of the DVAs Type 1 on the $1 / 3^{r d}$ octave bands is difficult to notice, the effect can clearly be seen in the better resolved $1 / 24^{\text {th }}$ octave bands spectra. A sharp improvement in the noise reduction index of about $10 \mathrm{~dB}$ occurs at a frequency of $320 \mathrm{~Hz}$, in case of 100 DVAs. The tested configurations with a lower number of DVAs turn out not be effective for counteracting the ETICS dip which can be explained by the limited span of control of the DVAs. At least 100 DVAs (spacing $\left\{d_{x}, d_{y}\right\}=0.32 \mathrm{~m}$ ) Type 1 are needed in order to obtain a significant effect, which is in agreement with the requirement stated in the theoretical Section 3.2.

The measured sound reduction index results $R$ for the Sorbothane-based Type 2 DVAs are shown in Fig. 33 and 34. Due to their higher damping ratio, the Sorbothanebased DVAs have a more broad-band effect as compared to the EPS based DVAs Type 1. Again, at least 100 DVAs were required to obtain a significant increase of the sound reduction index, in agreement with the numerical models presented in Section 3.2. Using 100 DVAs with a seismic mass of 410 grams (Type $2 \mathrm{a}$ ) increases the sound reduction index in the frequency range from $200-300 \mathrm{~Hz}$ by about 5 - $7 \mathrm{~dB}$. Using a seismic mass of 230 grams (Type 2b, $m_{D V A}=0.230 \mathrm{~kg}, \mu_{s c}=0.6 \%, \mu_{m l}=22 \%$ ) the improvement shifts this frequency range up to $240-350 \mathrm{~Hz}$. This shift in frequency is in reasonable accordance with the ratio $\sqrt{410 / 230}=1.34$. In the frequency range from 220 to $285 \mathrm{~Hz}$ improvements of the sound reduction index $R$ ranging from 7-10 dB were obtained for 100 DVAs Type $2 \mathrm{~b}$ (see Fig. 34).

\begin{tabular}{|l|l|l|l|}
\hline Configuration & $R_{w}$ & $\mathrm{C}$ & $\mathrm{C}_{t r}$ \\
\hline wall & 53.4 & -1.8 & -5.7 \\
wall+ETICS & 52.2 & -2.5 & -6.3 \\
wall+ETICS + 25 DVAs, Type 1 & 52.3 & -2.1 & -6.0 \\
wall+ETICS + 50 DVAs, Type 1 & 52.5 & -2.3 & -6.4 \\
wall+ETICS + 100 DVAs, Type 1 & 52.6 & -2.3 & -6.5 \\
\hline
\end{tabular}

Table 5: Single number quantities, EPS based DVAs (Type 1) 


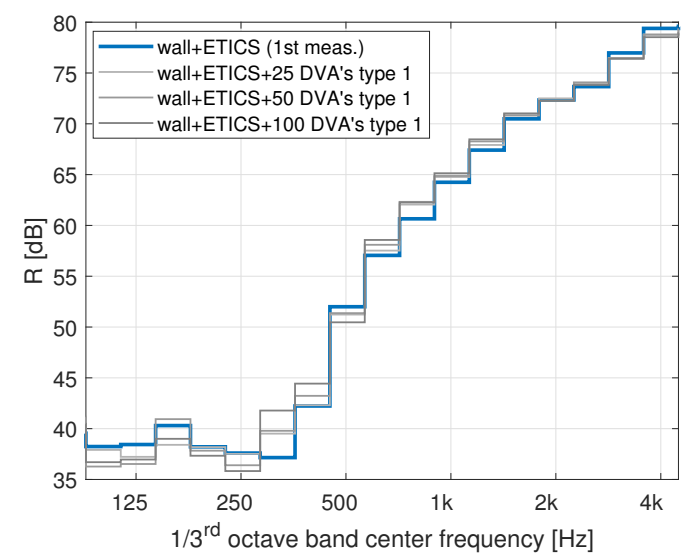

(a) Full frequency range

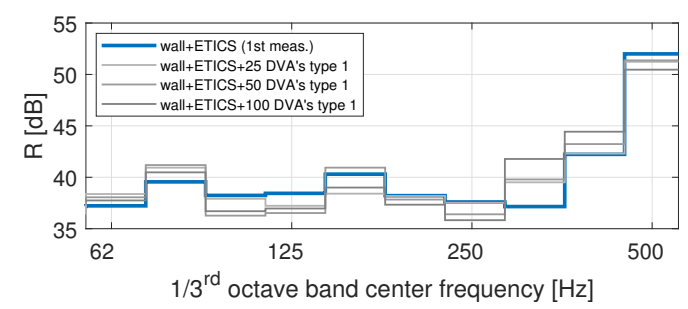

(b) Zoomed frequency range

Figure 31: Sound reduction index $R$, EPS based DVAs (Type 1), in $1 / 3^{r d}$ octave bands.

\begin{tabular}{|l|l|l|l|}
\hline Configuration & $R_{w}$ & $\mathrm{C}$ & $\mathrm{C}_{t r}$ \\
\hline wall & 53.4 & -1.8 & -5.7 \\
wall+ETICS & 52.2 & -2.5 & -6.3 \\
+ 25 DVAs, 2a & $52.1(52.1)$ & $-2.3(-2.3)$ & $-5.9(-5.9)$ \\
+ 50 DVAs, 2a & $53.2(53.4)$ & $-2.2(-2.3)$ & $-5.9(-6.0)$ \\
+ 100 DVAs, 2a & $53.9(54.3)$ & $-2.1(-2.4)$ & $-5.8(-6.0)$ \\
+ 100 DVAs, 2b & $53.5(54.1)$ & $-1.8(-2.0)$ & $-5.7(-5.8)$ \\
\hline
\end{tabular}

Table 6: Single number quantities, Sorbothane based DVAs (Type 2). Between brackets: corrected for sound radiation of DVAs.

Table 5 and Table 6 present the single number quantities (SNQs) $R_{W}, R_{W}+C$ and $R_{W}+C_{t r}$ for the bare wall and for the ETICS wall with and without DVAs. The SNQs were determined according to the ISO717-1 standard.

Concerning the uncertainty of the $R_{W}$ values in absolute sense, different works are available in literature for laboratory and field measurements. According to [41], the repeatability of the tests for a series of walls with almost the same mass per unit area of the one considered here, is $0.2 \mathrm{~dB}$. An evaluation of the uncertainty was made by Mahn and Pearse in [42]. These authors considered more than 200 building elements and found, for walls having a mass per unit area like the ETICS wall considered here, an uncertainty around $2.5 \mathrm{~dB}$. The uncertainty of the measured $R_{W}$ values in relative sense, for instance the measured $R_{W}$ for the ETICS wall with DVAs as compared to the measured $R_{W}$ for the ETICS wall without DVAs, is expected to be much more accurate. Repeated measure-

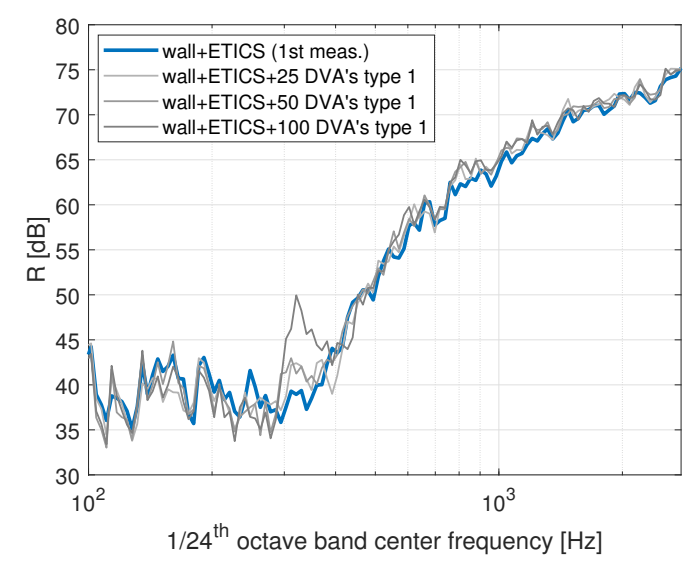

(a) Full frequency range

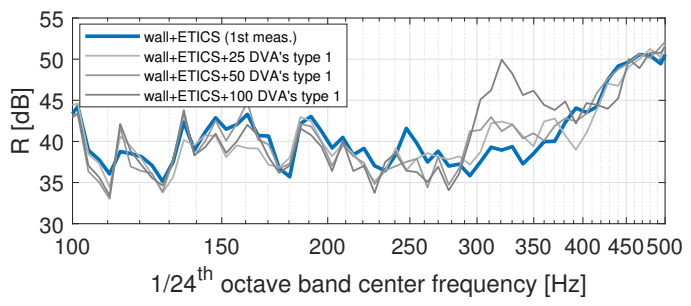

(b) Zoomed frequency range

Figure 32: Sound reduction index $R$, EPS based DVAs (Type 1), in $1 / 24^{\text {th }}$ octave bands.

ments on the same wall yielded results that are within 0.1 - $0.2 \mathrm{~dB}$ accuracy, which is in agreement with the uncertainty on the repeatability reported in [41]. The results between different wall configurations often differed less than $1 \mathrm{~dB}$. In order to allow easier comparisons between the results, the reference curve has been shifted by $0.1 \mathrm{~dB}$ steps and the $R_{W}$ values were expressed using one decimal place. This is not in compliance with the recommendation of the ISO 717-1 standard to present the SNQs as a full figure (no decimal places) is required for certification purposes. However, in the context of this work, it seems justified to allow a relative comparison more easily.

For the sake of prediction of further optimized DVA configurations, using the spatially resolved LDV-measurement data discussed in Section 5.1 (Fig. 30c), we have also simulated the situation where the DVAs would be incorporated in the EPS layer, so as not to contribute to acoustic radiation (see also Liu et al. [17]). The resulting values for the SNQs are shown in brackets in Table 6 for DVAs Type 2. Improvements range from $0.1 \mathrm{~dB}$ to $0.6 \mathrm{~dB}$. .

Figures 35 and 36 give a graphical representation of the single number quantities. For the SNQs of DVA Type 2, both the SNQs that follow directly from the sound reduction index measurements, as well as the corrected measurement data to eliminate the radiation of the DVA seismic masses are shown.

From the tables and from the figures it can be concluded that for the EPS based DVAs, $R_{W}$ only slightly increased from $52.2 \mathrm{~dB}$ (ETICS wall) to $52.6 \mathrm{~dB}$ (100 DVAs, Type 


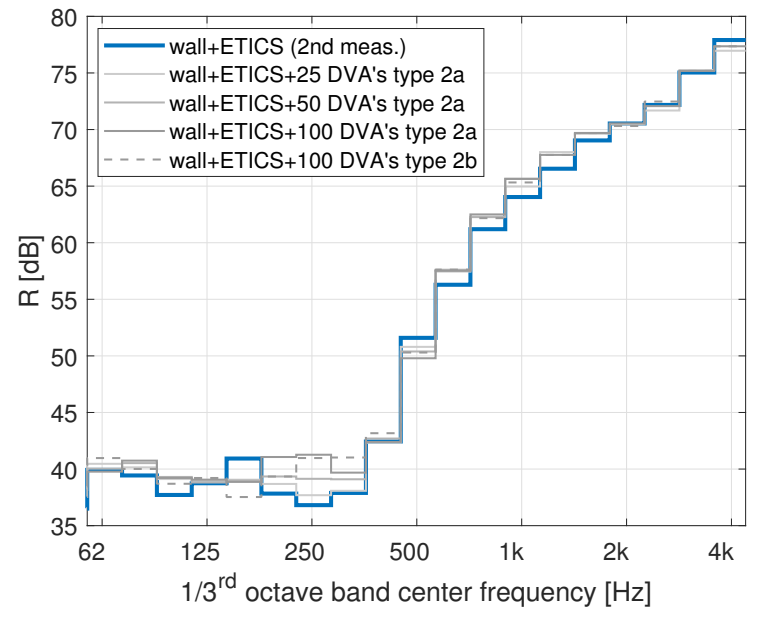

(a) Full frequency range, as measured.

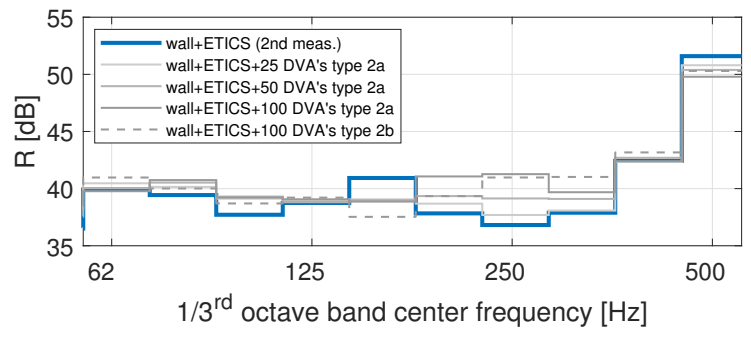

(b) Zoomed frequency range, as measured.

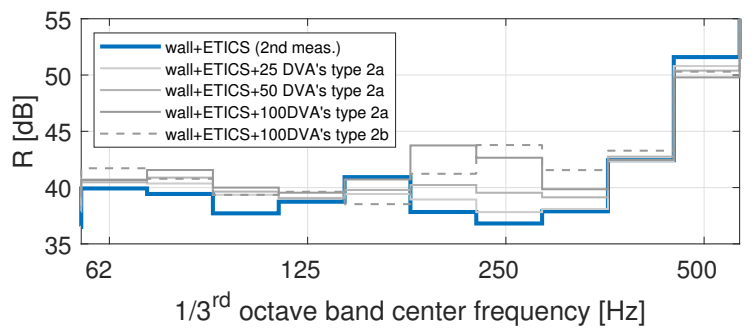

(c) Zoomed frequency range, LDV corrected for sound radiation DVA seismic mass.

Figure 33: Sound reduction index $R(f)$, Sorbothane based DVAs (Type 2 ), in $1 / 3^{\text {rd }}$ octave bands.

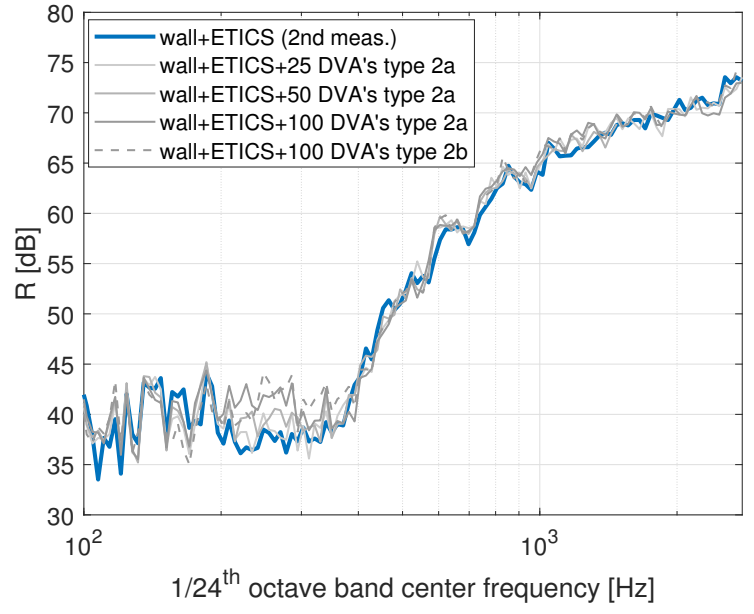

(a) Full frequency range, as measured.

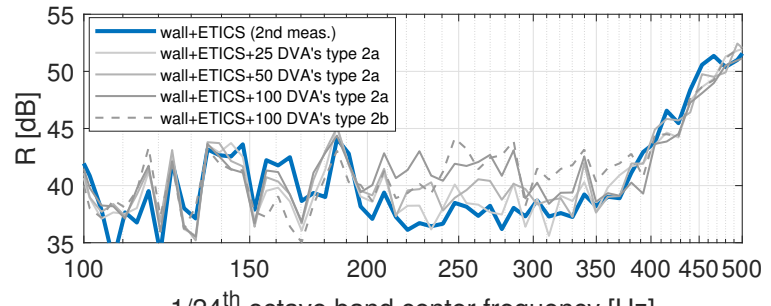

$1 / 24^{\text {th }}$ octave band center frequency $[\mathrm{Hz}]$

(b) Zoomed frequency range, as measured.

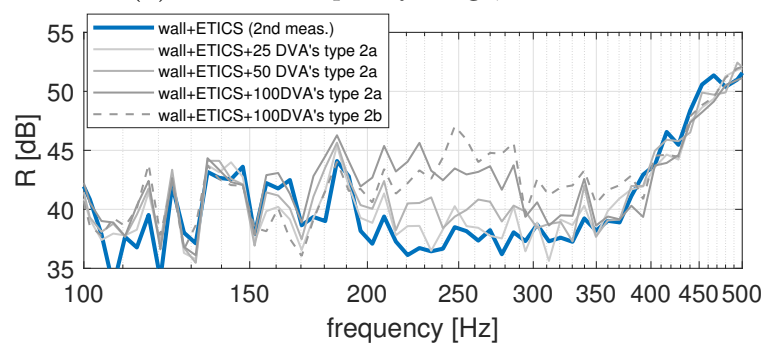

(c) Zoomed frequency range, LDV corrected for sound radiation DVA seismic mass.

Figure 34: Sound reduction index $R$, Sorbothane based DVAs (Type 2 ), in $1 / 24^{\text {th }}$ octave bands. 


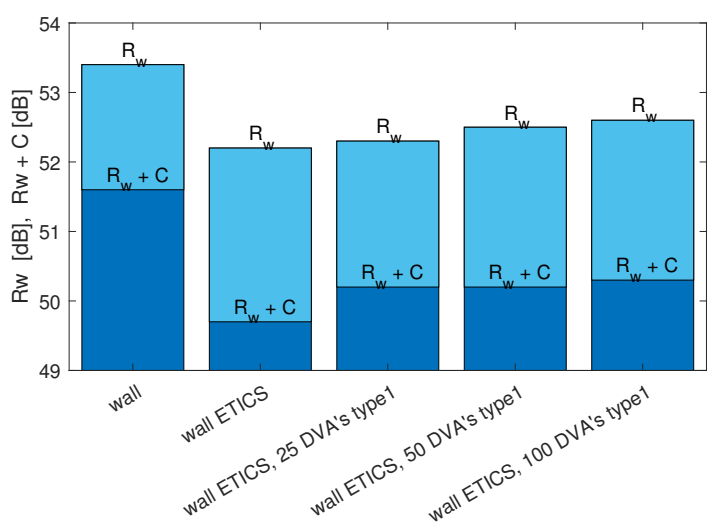

(a) EPS based DVAs Type 1

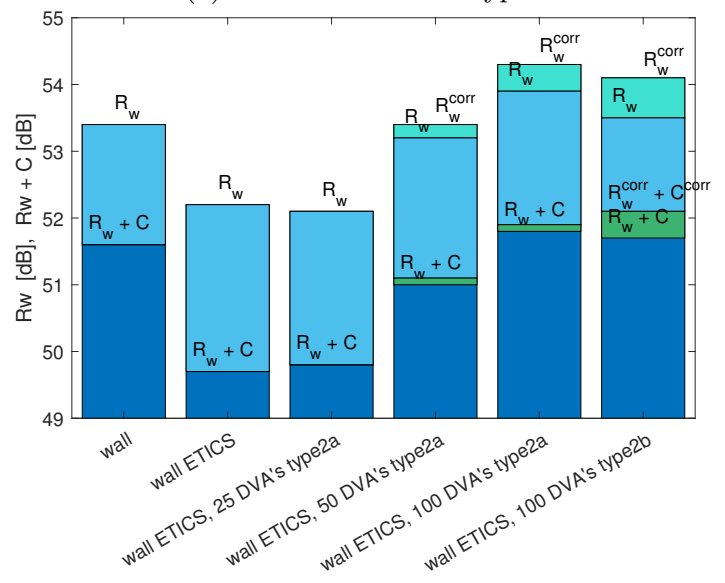

(b) Sorbothane based DVAs Type 2. Non-corrected SNQs shown in dark and light blue. Corrections for the sound radiation of the DVAs shown in green and turquoise.

Figure 35: Single numer quantities $R_{w}$ and $R_{w}+C$.

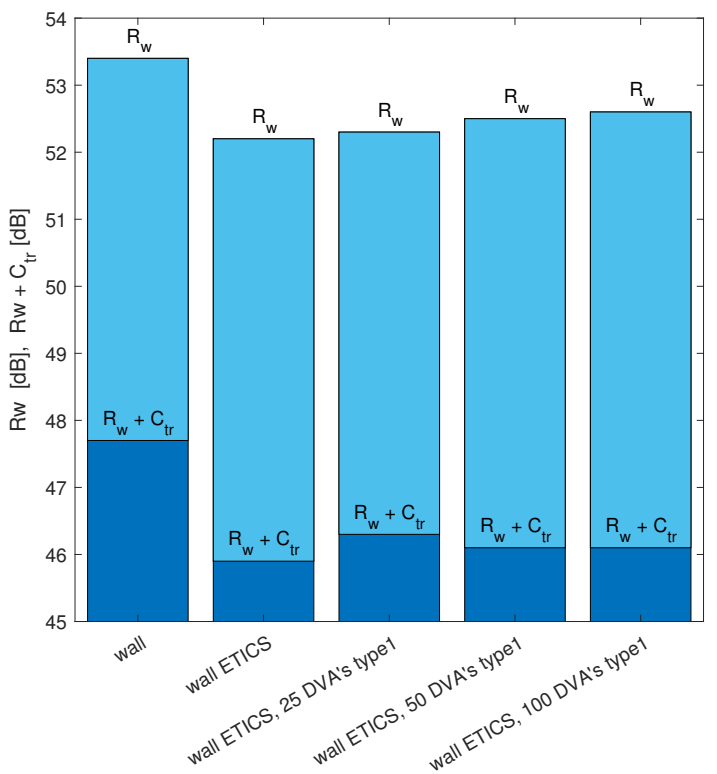

(a) EPS based DVAs Type 1

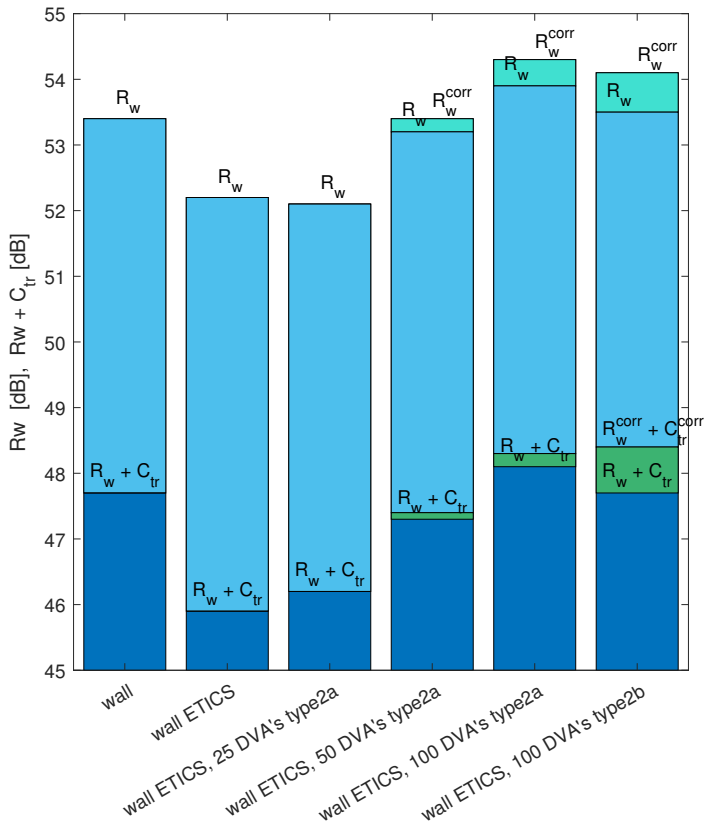

(b) Sorbothane based DVAs Type 2. Non-corrected SNQs shown in dark and light blue. Corrections for the sound radiation of the DVAs shown in green and turquoise.

Figure 36: Single numer quantities $R_{w}$ and $R_{w}+C_{t r}$. 
$1)$.

For the Sorbothane-based DVAs, $R_{W}$ increased from $52.2 \mathrm{~dB}$ (ETICS wall) to $53.9 \mathrm{~dB}$ (100 DVAs, Type 2a), corresponding with an improvement of $1.7 \mathrm{~dB}$. After correcting for the sound radiation of the DVA seismic masses, this difference goes up to $2.1 \mathrm{~dB}(54.3-52.2 \mathrm{~dB})$. In effect, the ETICS wall with DVAs shows a better performance in $R_{W}$ as compared to the bare wall $(53.4 \mathrm{~dB})$.

Summarizing, when considering the corrections for traffic noise, the $R_{W}+C_{t r}$ difference between the ETICS wall and the ETICS wall with DVAs Type 2a is $2.2 \mathrm{~dB}$ and $2.4 \mathrm{~dB}$, for the standard and for the sound radiation corrected DVAs respectively. Considering the $1 / 12^{\text {th }}$ octave spectrum, the sound reduction index $R$ was improved by $7-10 \mathrm{~dB}$ in the frequency range from 220 to $285 \mathrm{~Hz}$ (see Fig. 34) using 100 DVAs Type $2 \mathrm{~b}\left(m_{D V A}=0.230 \mathrm{~kg}, \mu_{s c}\right.$ $=0.6 \%, \mu_{m l}=22 \%$ ).

\section{Conclusions}

The article has addressed the problem of the loss of acoustic performances related to the application of ETICS systems to façade walls. While having beneficial effects for the thermal insulation and energy performance of buildings, the use of ETICS gives issues from the acoustic insulation point of view, due to a structure related massspring-mass resonance in the low frequency range. The vibro-acoustic effect of the additional thermal insulation and finishing layer was analysed by using a finite element model and applying Bloch-Floquet boundary conditions in order to solve efficiently the problem.

The use of dynamic vibration absorbers to mitigate the ETICS induced dip in the acoustic isolation curve was assessed by simulations and experiments for different design configurations. It was numerically shown that DVAs mounted on the layer of reinforced adhesive mortar have a limited span of control due to the finite stiffness of this element. This effect sets a maximum to the inter-distance of the DVAs and thus a minimum on the number of DVAs needed. As expected, the heavier the seismic mass of the DVA, the larger the effect on the sound reduction index $R$.

Three types of DVAs were mounted in different amounts on a real scale ETICS wall, built in a sound transmission suite facility. Because of the limited span of control of the DVAs, the measurements showed that a minimum number of 100 DVAs for an area of about $10 \mathrm{~m}^{2}$ is required to achieve reductions from 7 to $10 \mathrm{~dB}$ in terms of the sound reduction index at the ETICS mass-spring-mass resonance frequency, at the expense of a mass increase of only $0.6 \%$ $\left(\mu_{s c}\right)$ relative to the weight of the silicate cement brick wall. In terms of the single number quantity, $R_{W}$ was improved by about $2 \mathrm{~dB}$.

Spatially resolved Laser Doppler vibration measurements showed that at specific frequencies a reduction of the spatially averaged vibration level of about $\Delta L_{V}=10 \mathrm{~dB}$ can be obtained. These meaurements also showed that the application of DVAs to improve the acoustic performance of ETICS systems can be further optimized by embedding the DVAs in between the thermal insulation and the layer of reinforced adhesive mortar, thus blocking their sound radiation into the air. As a result, further $R_{W}$ reductions up to $0.6 \mathrm{~dB}$ are possible.

\section{Acknowledgments}

The project was carried out in the framework of H2020MSCA-RISE-2015 No. 690970 "Papabuild". Company A\&Z Acoustics s.r.o., Repašského 2, 84102 Bratislava, is acknowledged for the financial support of DU. The authors appreciate Knauf, Engis, Belgium, for supplying materials for ETICS installation. The authors are grateful to Cynthia Micallef, Yannick Sluyts, Peilong Yuan, Werner Neefs and Bernd Salaets of KU Leuven for their kind help, and Piet Vanmeenen for building the ETICS system.

DU acknowledges the support of the International Mobility of Researchers call in the framework of the Operational Programme Research, Development and Education, project reg. num. CZ.02.2.69/0.0/0.0/16_027/0008465.

\section{References}

[1] International organization for standardization iso 12354-1: Building acoustics - estimation of acoustic performance of buildings from the performance of elements - part 1: Airborne sound insulation between rooms., Standard (2017).

[2] K. Miskinis, V. Dikavicius, A. Buska, K. Banionis, Influence of EPS, mineral wool and plaster layers on sound and thermal insulation of a wall: a case study, Applied Acoustics 137 (2018) 62-68. doi:10.1016/j.apacoust.2018.03.001.

[3] A. Santoni, P. Bonfiglio, J. L. Davy, P. Fausti, F. Pompoli, L. Pagnoncelli, Sound transmission loss of ETICS cladding systems considering the structure-borne transmission via the mechanical fixings: Numerical prediction model and experimental evaluation, Applied Acoustics 122 (2017) 88-97. doi:10.1016/j.apacoust.2017.02.008.

[4] D. Urbán, P. Zat'ko, N.B. Roozen, H. Muellner, C. Glorieux, Influence of the dynamic stiffness of external thermal insulation on the sound insulation of walls, Euronoise 2018, Crete (2018).

[5] D. Urbán, N.B. Roozen, O. Jiricek, Influence of the dynamic stiffness of external thermal insulation on the sound insulation of walls, Internoise 2019, Madrid (2019).

[6] D. Urbán, N.B. Roozen, H. Muellner, P. Zaťko, A. Niemczanowski, M. Rychtáriková, C. Glorieux, Vibrometry Assessment of the External Thermal Composite Insulation Systems Influence on the Façade Airborne Sound Insulation †, Applied Sciences 8 (5) (2018) 703. doi:10.3390/app8050703.

[7] P. Gardonio, E. Turco, Tuning of vibration absorbers and Helmholtz resonators based on modal density/overlap parameters of distributed mechanical and acoustic systems, Journal of Sound and Vibration 451 (2019) 32-70. doi:10.1016/j.jsv.2019.03.015.

[8] E. Waterman, D. Kaptein, S. Sarin, Fokker's activities in cabin noise control for propeller aircraft, in: SAE Technical Paper 830736, 1983, 1983.

[9] C. Droz, O. Robin, M. Ichchou, N. Atalla, Improving sound transmission loss at ring frequency of a curved panel using tunable 3D-printed small-scale resonators, The Journal of the Acoustical Society of America 145 (1) (2019) EL72-EL78. doi:10.1121/1.5088036. 
[10] J. Jung, H.-G. Kim, S. Goo, K.-J. Chang, S. Wang, Realisation of a locally resonant metamaterial on the automobile panel structure to reduce noise radiation, Mechanical Systems and Signal Processing 122 (2019) 206-231. doi:10.1016/j.ymssp.2018.11.050.

[11] J. Ormondroyd, J. Den Hartog, The theory of the dynamic vibration absorber, J. Appl. Mech. 50 (1928).

[12] D. J. Thompson, The theory of a continuous damped vibration absorber to reduce broad-band wave propagation in beams (2017) ISVR Technical Memorandum No 968, 2017.

[13] D. Thompson, A continuous damped vibration absorber to reduce broad-band wave propagation in beams, Journal of Sound and Vibration 311 (3-5) (2008) 824-842. doi:10.1016/j.jsv.2007.09.038.

[14] L. Van Belle, C. Claeys, E. Deckers, W. Desmet, The impact of damping on the sound transmission loss of locally resonant metamaterial plates, Journal of Sound and Vibration 461 (2019) 114909. doi:10.1016/j.jsv.2019.114909.

15] N. de Melo Filho, C. Claeys, E. Deckers, W. Desmet, Realisation of a thermoformed vibro-acoustic metamaterial for increased STL in acoustic resonance driven environments, Applied Acoustics 156 (2019) 78-82. doi:10.1016/j.apacoust.2019.07.007.

[16] M. Jovanoska, Overcoming the coincidence effect of a single panel by introducing and tuning locally resonant structures, in: Proceedings of INTERNOISE 2019, Madrid, 2019.

[17] Z. Liu, R. Rumpler, L. Feng, Broadband locally resonant metamaterial sandwich plate for improved noise insulation in the coincidence region, Composite Structures 200 (2018) 165-172. doi:10.1016/j.compstruct.2018.05.033.

[18] International organization for standardization iso 10140: Acoustics. laboratory measurement of sound insulation of building elements - part 2: Measurement of airborne sound insulation and part 4: Measurement procedures and requirements, 2010., Standard (2010).

[19] F. Bloch, Über die Quantenmechanik der Elektronen in Kristallgittern, Z. Phys. A, Hadrons Nucl. 52 (1929) 555-600.

[20] P. Langlet, A. Hladkyhennion, J. Decarpigny, Analysis of the propagation of plane acoustic-waves in passive periodic materials using the finite-element method, Journal of the Acoustical Society of America 98 (5, 1) (1995) 2792-2800. doi:10.1121/1.413244.

[21] L. Brillouin, Wave Propagation in Periodic Structures, McGraw-Hill Book Company, 1946.

22] M. I. Hussein, Reduced Bloch mode expansion for periodic media band structure calculations, Proceedings of the Royal Society A - Mathematical Physical and Engineering Sciences 465 (2109) (2009) 2825-2848. doi:10.1098/rspa.2008.0471.

[23] U. Arasan, F. Marchetti, F. Chevillotte, G. Tanner, D. Chronopoulos, E. Gourdon, On the accuracy limits of plate theories for vibro-acoustic predictions, Journal of Sound and Vibration (2020). doi:https://doi.org/10.1016/j.jsv.2020.115848.

[24] D. A. Bies, C. H. Hansen, Engineering Noise Control, 4th Edition, Spon, 2009.

[25] N.B. Roozen, L. Labelle, C. Glorieux, On the unwrapping of dispersion curves in the irreducible Brillouin zone by means of a spatial Fourier transform approach, International Journal of Solids and Structures 196-197 (2020) 67-75. doi:10.1016/j.ijsolstr.2020.03.016.

[26] F. Marchetti, K. Ege, Q. Leclère, N.B. Roozen, On the structural dynamics of laminated composite plates and sandwich structures; a new perspective on damping identification, Journal of Sound and Vibration 474 (2020) 115256. doi:10.1016/j.jsv.2020.115256.

[27] N.B. Roozen, L. Labelle, M. Rychtáriková, C. Glorieux, Determining radiated sound power of building structures by means of laser Doppler vibrometry, Journal of Sound and Vibration 346 (2015) 81-99. doi:10.1016/j.jsv.2015.02.029.

[28] N.B. Roozen, Q. Leclère, D. Urbán, L. Kritly, C. Glorieux, Assessment of the sound reduction index of building elements by near field excitation through an array of loudspeakers and structural response measurements by laser
Doppler vibrometry, Applied Acoustics 140 (2018) 225-235. doi:10.1016/j.apacoust.2018.06.002.

[29] M. Heckl, Radiation from plane sound sources. [abstrahlung von ebenen schallquellen.], Acustica 37 (3) (1977) 155-166, cited By 19.

[30] E. G. Williams, J. D. Maynard, Numerical evaluation of the Rayleigh integral for planar radiators using the FFT, The Journal of the Acoustical Society of America 72 (6) (1982) 20202030. doi:10.1121/1.388633.

[31] E. G. Williams, J. A. Mann, Fourier Acoustics: Sound Radiation and Nearfield Acoustical Holography, The Journal of the Acoustical Society of America 108 (4) (2000) 1373-1373. doi:10.1121/1.1289662

[32] F. Fahy, Sound and structural vibration; Radiation, transmission and response, Academic press, 1994.

[33] A. C. Eringen, E. S. Suhubi, Elastodynamics, Academic press, 1975.

[34] C. Hopkins, Sound Insulation, Butterworth-Heinemann, Elsevier, 2007

[35] M. Zilletti, S. J. Elliott, E. Rustighi, Optimisation of dynamic vibration absorbers to minimise kinetic energy and maximise internal power dissipation, Journal of Sound and Vibration 331 (18) (2012) 4093-4100. doi:10.1016/j.jsv.2012.04.023.

[36] D. Ewins, Modal testing: theory and practice, Research Studies Press LTD, 1986.

[37] J. Bendat, A. Piersol, Engineering Applications of Correlation and Spectral Analysis, Wiley-Interscience, NewYork, 1980.

[38] Q. Leclere, Multi-channel spectral analysis of multi-pass acquisition measurements, Mechanical Systems and Signal Processing 23 (2009) 1415-1422. doi:10.1016/j.ymssp.2008.12.002.

[39] N.B. Roozen, Q. Leclère, M. Rychtáriková, C. Glorieux, A global error estimator for the uncertainty of a multichannel spectral analysis, Applied Acoustics 87 (2014) 57-63. doi:10.1016/j.apacoust.2014.06.007.

[40] P. K. Banerjee, R. Butterfield, Boundary element methods in engineering science, McGraw-Hill, London, 1981.

[41] C. Scrosati, F. Scamoni, M. Bassanino, M. Mussin, G. Zambon, Uncertainty analysis by a Round Robin Test of field measurements of sound insulation in buildings: Single numbers and low frequency bands evaluation - Airborne sound insulation, Noise Control Engineering Journal 61 (3) (2013) 291-306. doi: $10.3397 / 1 / 3761025$

[42] J. Mahn, J. Pearse, The Uncertainty of the Proposed Single Number Ratings for Airborne Sound Insulation, Building Acoustics 19 (3) (2012) 145-172. doi:10.1260/1351010X.19.3.145. 